\title{
Ook Lideg
}

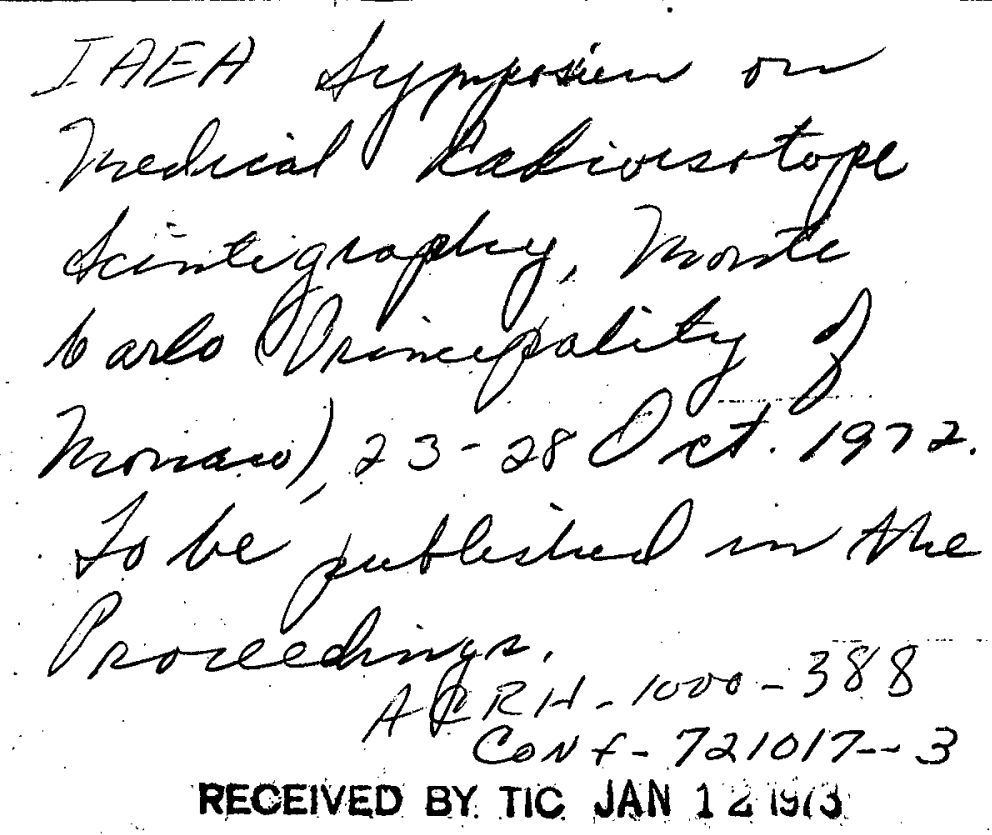

ADVANCE IN FUNDAMENTAL ASPECTS OF IMAGING SYSTEMS AND TECHNIQUES

R. N. Beck, L. T. Zinmer, D. B. Charleston, P. V. Harper
and P. B. Hoffer

Department of Radiology, University of Chicago and Argonne Cancer Research Hospital*

Chicago, Illinois U.S.A.

ABSTRACT

An analysis of the radionuclide imaging process is presented in an outline of the relationships between parameters describing the object, radiation detector system, numerical image, photo-recording system, and exposure image, assuming that the imaging system is linear and shift-invariant. Major image imperfections such as quantum noise and linear distortion are described quantitatively in terms of the sensitivity and spatial resolution of the detector and recording systems. Principles of system design are formulated: the maximum useful detector sensitivity is limited by the size of the smallest structures to be detected; the maximum useful recorder sensitivity is limited by the required signal-to-noise ratio $(S / N)$ or confidence of detection. A theory of optimum-weighted multichannel imaging is described which permits maximization of $S / N$, utilizing the entire pulse amplitude spectrum. The effects of image processing on noise in the image are described quantitatively: smoothing tends to reduce image noise and increase linear distortion; sharpening tends to have the opposite effects. The use of a reconding mediun capable of a bipolar response (repeated local storage and erasure) permits on-line image sharpening or reduction of linear distortion that is introduced by the detector system (which is described as a low-pass filter of spatial frequency components comprising the object). The need for a more general formulation of the imaging process, including the effects of nonlinear and nonstationary components on diagnostic image quality, is emphasized. The use of Receiver Operating Characteristic (ROC) curves is suggested as a means for comparing and evaluating diagnostic image quality in terms of the accuracy of the physician's performance in interpreting radionuclide images. The general principles, concepts, and terms used to describe and evaluate radionuclide imaging systems can be adapted to other imaging modes used in nuclear medicine. 


\section{DISCLAIMER}

This report was prepared as an account of work sponsored by an agency of the United States Government. Neither the United States Government nor any agency Thereof, nor any of their employees, makes any warranty, express or implied, or assumes any legal liability or responsibility for the accuracy, completeness, or usefulness of any information, apparatus, product, or process disclosed, or represents that its use would not infringe privately owned rights. Reference herein to any specific commercial product, process, or service by trade name, trademark, manufacturer, or otherwise does not necessarily constitute or imply its endorsement, recommendation, or favoring by the United States Government or any agency thereof. The views and opinions of authors expressed herein do not necessarily state or reflect those of the United States Government or any agency thereof. 


\section{DISCLAIMER}

Portions of this document may be illegible in electronic image products. Images are produced from the best available original document. 


\section{ADVANCES IN FUNDAMENTAL ASPECTS OF IMAGING SYSTEMS AND TECHNIQUES}

R. N. Beck, L. T. Zinmer, D. B. Charleston P. V. Harper, and P. B. Hoffer

Department of Radiology, University of Chicago and Argonne Cancer Research Hospital* Chicago, Illinois U.S.A.

\section{INTRODUCTION}

The types of imaging processes currently used in nuclear medicine constitute only a small fraction of all imaging processes; nevertheless, the number of categories required to classify them is astonishing. In general, image formation involves a source of radiation, a radiation detector, and a recording devi.ce. Imaging modes are typically described in terms that characterize one or more properties of these elements.

The radiation used for imaging in nuclear medicine may consist of photons that are emitted from, transmitted through, stimulated within, or scattered by an object. Thus, the object itself can be thought of as a three-dimensional spatial distribution of material, each point of which has a certain self-radiance, transmittance, fluorescence, or scattering coefficient. In addition, the objects of interest in nuclear medicine are surrounded by or embedded in the patient's tissue, a medium that scatters and absorbs some of the radiation used to characterize the object. In general, we can distinguish between imaging modes that utilize radiation from in internal source and those that require an external source.

The most widely used imaging mode is that in which the object to be imaged is the radioactivity distributed within an ongan. Since the radiation utilized in this process is emitted spontaneously by the radioactive material, we think of this as an emission imaging mode.

An important variation on this theme is the coincidence mode $[1,2]$, used to image the distribution of a positron emitter by detecting the associated annihilation photons in coincidence.

The incoherent holographic imaging mode $[3,4]$ deserves special mention as a most recent and promising variation on the emission imaging theme. In this case, a pseudo-hologram is formed with incoherent radiation

\footnotetext{
Operated by the University of Chicago for the United States Atomic Energy Commission.
} 
from the radioactive object; the 3-D image is then projected with a coherent light beam as in ordinary holography.

These modes can be distinguished from the transmission imaging mode $[5,6,7,8]$, in which a shadow image is formed of internal structures having different attenuation values for the external source of radiation.

In the fluorescence imaging mode $[9,10,11,12]$, an external source of radiation is used to stimulate the emission of X-rays by a stable high- $\mathrm{Z}$ material distributed within the patient. An image of this distribution is formed by the detection of the fluorescent X-rays.

In some respects, this is similar to the scatter imaging mode [13], in which radiation from an external source is scattered differentially by intermal structures having different compton scattering coefficients. An image of these structures is formed by the detection of the scattered radiation.

The detector for a particular mode may utilize one on more scintillators, semiconductors, wine chambers, or fluorescent screens, depending on the requirements. Usually, the imaging mode can be implemented by detectors which may be movable or stationary; that is, by a scanner, a camera, or a hybrid system, based on the prototypes of Cassen [14], Anger [15], and Harper [16], respectively. In addition, each mode can in principle be implemented to provide static or dynamic images as well as various forms of tomographic projection.

The reconding of detected radiation can be performed by analog on digital means for immediate analysis or display, or for further processing.

our goal is to understand these processes well enough so that we can design imaging systems that will provide diagnostically useful information, while minimizing the radiation dosage, the inconvenience, and the stress to the patient and hospital staff.

In the absence of specific knowledge as to which object features would provide the most useful information, an ideal imaging system would provide an exact replica of the object. Although some advances have been made toward this ideal, existing systems produce images that are imperfect in many ways. Here, we shall briefly summarize a theoretical analysis of the imaging process in terms that will elucidate the nature and origins of the major imperfections. Thus, we hope to provide a framework in which to fit recent advances, and a rationale for future research.

\section{ANALYSIS OF AN IMAGING PROCESS}

The first fundamental question to be answered in the analysis of any imaging process is: "What is the image of a typical object element?" The answer involves, first, an identification of suitable typical object elements, from which any real object can be synthesized; second, a description of the response of the radiation detector system to a typical element; and thind, a description of the response of the recording system to a typical input from the detector. The image, then, consists of an aggregate of imaged object elements.

In any theoretical formulation, nomenclature is always a problem. It is probably accurate to say that there is little serious disagreement concerning most of the concepts that may be used to analyze the imaging processes; there is little agneement, however, on the terms used to describe these concepts, and even less agreement on the symbols used to designate these terms. This state of affairs must be expected in any field that is developing rapidly on many fronts and making free use of nomenclature from related fields, such as communications, optics, and radiography, in which consistency has not yet been fully achieved. Departures from previously 
used concepts, terms, and symbols are sufficiently numerous and subtle to warrant a brief summary of one form that the analysis might take at this point in its evolution. No suggestion of finality is intended. A glossary of the most important terms and symbols appears in Appendix A.

In the discussion to follow, an analysis of the emission mode of imaging with scintillation detectors and photographic film as the recording medium will be used to illustrate an approach that can be adapted to other modes.

\subsection{The object}

In an analysis of the emission mode, the object to: be imaged can be thought of as consisting of elements which may be described in various ways. For example, a three-dimensional distribution of radioactivity can be described by

$$
\rho\left(\vec{r}_{0} ; E\right) \equiv \rho\left(\mathrm{x}_{\mathrm{O}}, \mathrm{y}_{\mathrm{O}} ; \mathrm{z}_{\mathrm{O}} ; \mathrm{E}\right)
$$

[the expected number of photons with energy $E$ emitted at $\vec{r}_{0} / \mathrm{sec}-\mathrm{cm}^{3}$ ] which is proportional to the concentration of radioactivity at each point $\vec{r}_{0}$ in object space. ${ }^{1}$ This object is embedded in a medium (the patient's tissues) having an attenuation coefficient $\mu\left(\vec{r}_{0} ; E\right)\left[\mathrm{cm}^{-1}\right]$. For simplicity, we shall assume that the medium is uniform.

All other descriptions of the object can be expressed in terms of $\rho$. For example, the object may be regarded as a set of point sources with intensity

$$
\rho\left(\vec{r}_{0} ; E\right) d \vec{r}_{0}[\text { photons/sec }]
$$

where

$$
\overrightarrow{d r}_{0} \equiv d x_{0} d y_{0} d z_{0} \text {. }
$$

Since the sensitivity and spatial resolution of detector systems tend to be constant over planes located at a fixed distance from the detector, it is most convenient to think of the object as a set of thin sheets perpendicular to the detector axis, on each of which the intensity on concentration at every point can be described by a two-dimensional function of the transverse coordinates $x_{0}$ and $y_{0}$, with parameters $z_{0}$ and $E$; that is,

$$
\rho\left(\mathrm{x}_{\mathrm{O}}, \mathrm{y}_{\mathrm{O}} ; \mathrm{z}_{\mathrm{O}}, \mathrm{E}\right) \mathrm{d} \mathrm{z}_{0} \text { [photons emitted at } \vec{r}_{0} / \mathrm{sec}-\mathrm{cm}^{2} \text { ]. }
$$

The image of $\rho$ projected onto a plane is then the sum of images of planar objects at all distances $z_{0}$.

To simplify the notation, the symbols

$$
o\left(x_{0}\right) \equiv \rho\left(x_{0} ; z_{0}, E\right) d z_{0}
$$

${ }^{1}$ Although the spatial distribution of radioactive atoms is discontinuous on a distance scale of atomic dimensions, and the decay process is discontinuous, random, and decreasing with respect to time, $\rho$ is a continuous function in space, nonrandom, and constant in time, from which we can compute the expected image. The effects of the discontinuous and random nature of radioactive decay on the image will be treated later as separate
issues. 
and

$$
o\left(r_{0}\right) \equiv o\left(x_{0}, y_{0}\right) \equiv \rho\left(x_{0}, y_{0} ; z_{0}, E\right) d z_{0}
$$

will be used to designate these planar objects when the intensity varies on one and two transverse dimensions, respectively.

The total photon emission per second from the object $o\left(r_{0}\right)$. [photons emitted at $\left.r_{0} / \mathrm{sec}-\mathrm{cm}^{2}\right]$ is just

$$
\int_{-\infty}^{\infty} \int_{0} o\left(r_{0}\right) d r_{0} \equiv 0_{0}[\text { photons emitted/sec]. }
$$

If $A_{0}\left[\mathrm{~cm}^{2}\right]$ is the area of $o\left(r_{0}\right)$, beyond which $o\left(r_{0}\right)$ is zero, and

$\bar{O}$ [photons emitted $/ \mathrm{sec}-\mathrm{cm}^{2}$ ] is the average object intensity over this area, then $\odot_{0}=\bar{O} A_{0}$.

If we define the normalized object by $o^{\prime}\left(r_{0}\right) \equiv o\left(r_{0}\right) / \odot_{0}$, then the object can be described by the product of two factors that refer to its total intensity and shape, respectively; that is,

$$
O\left(r_{0}\right)=O_{0} O^{\prime}\left(r_{0}\right)=\bar{O} A_{0} O^{\prime}\left(r_{0}\right) \text {. }
$$

Typical elements of one- and two-dimensional planar objects may be regarded as uniform line sources and point sources, respectively. The fundamental theorem of Fourier analysis assures us, however, that an alternative description of the object is possible, since any such function of one or two dimensions that describes a real object can be regarded as a superposition or summation of plane waves consisting of sinusoidal variations in intensity. In either the one- or the two-dimensional case, the typical object element is just such a plane wave, with spatial frequency $v[$ cycles $/ \mathrm{cm}]$. (This is very. closely analogous to the practice of describing a complex sound pattern, such as music, in terms of the spectrum of sinusoidal components with temporal frequencies $f$ [cycles/second], of which it is composed. One form of this useful analogy between radionuclide imaging and sound reproduction is depicted more completely in Fig. I.)

The spatial frequency components comprising a planar object such as $o\left(r_{0}\right)$ are described by the unnormalized two-dimensional frequency spectrum $\theta(v) \equiv \theta(\vec{v})=\theta\left(v_{x}, v_{v}\right)$, which is the Fourier transform (F.T.), of the object; that is,

$$
O(v) \equiv \text { F.T. }\left[o\left(r_{0}\right)\right] \equiv \int_{-\infty}^{\infty} \int_{-\infty} o(x, y) e^{-j 2 \pi\left(v_{x} x+v_{y} y\right)} d x d y .
$$

From equation (1), the value of $\theta(v)$ at $v=0$ is just $\theta_{0}$. This is used to define the normalized object spectrum,

$$
\Theta^{\prime}(\nu) \equiv \frac{\Theta(\nu)}{\theta_{0}}=\frac{\theta(\nu)}{\bar{\partial} A_{0}},
$$

which has the value 1 at $v=0$.

In addition, the planar object can be formally synthesized from its spectrum by means of the inverse Fourier transform, F.T. ${ }^{-1}$; that is,

$$
o\left(r_{0}\right)=F \cdot T \cdot \cdot^{-1}[O(\nu)] \equiv \int_{-\infty}^{\infty} \int\left(\nu_{x}, \nu_{y}\right) e^{j 2 \pi\left(\nu_{x} x+\nu_{y} y\right)} d \nu_{x} d \nu_{y}
$$


Though perhaps less familiar than sound-reproducing systems, certain optical systems are more closely analogous to radionuclide imaging systems, and provide additional insight into the concepts and methods of image formation. For example, in optics, $O(v)$ is the Fraunhofer diffraction pattern of the object $o\left(r_{0}\right)$. This pattern may be regarded as a spatial display of the Fourier spectrum of plane wave components comprising $o\left(r_{0}\right)$. Some imaging operations in optics $[17,18,19]$, can best be understood as occurring in two stages, involving a Fourier transform of the object followed by an inverse Fourier transform of the object spectrum to produce an image.

It is helpful for understanding the incoherent holographic imaging mode $[3,4,19,20]$ to know that $o\left(r_{0}\right)$ may also be subjected to a Fresnel transform (in which each object point is transformed to a Fresnel diffraction patterm), and that a hologram is a reconding of the superposition of the Fresnel diffraction patterms of all points of the object. While this is usually achieved with coherent light, the Fresnel diffraction pattern can be recorded with incoherent radiation, such as $\gamma$-ray photons, by interposing a lead or gold replica of this pattern between the $\gamma$-ray source and the detector, thus casting a shadow of the pattern onto the detector for each point source comprising the object. The result is a pseudo-hologram of the object. The image is then projected in the conventional way, with coherent light.

In general, the most appropriate description of the object depends on the nature of the transformations performed by the imaging system in the image-forming operation.

\subsection{The detector system}

In any imaging system, the detector per se is that component with which radiation from the object interacts with sufficient transfer of energy to produce some useful output. To simplify the analysis, ${ }^{2}$ the detector system is thought of as including everything between the object and the recording device that intervenes in any way affecting the image. For the typical emission imaging mode, this includes the patient's tissue, the collimator, the detector per se, and the pulse height analyzer, as depicted in the lower portion of Fig. 2.

To form a satisfactory image of the object, the detector system must possess adequate sensitivity and spatial resolution. These properties are usually interdependent in an inverse manner, so that a satisfactory compromise must be achieved.

In addition, characteristics such as depth of field, energy resolution, temporal resolution or resolving time, linearity, and shift-invariance or stationarity, may have significant effects on the image quality as well as on the degree of difficulty encountered in the analysis of the imaging: process.

2The altemative approach would require a description of the "effective object," i.e., the radiation field emerging from the patient, which is more complex than a description of the distribution of radioactivity. 


\subsubsection{Components of response}

Radiation from the object may. enter the detector by three main

routes: (a) Photons may penetrate the overlying tissue without interacting and pass through the collimator properly, or "geometrically." (b) They may penetrate the collimator septa or shielding. (c) Alternatively, the photons may undergo Compton scattering interactions in the patient's tissues, with the scattered photons entering the detector. Thus, the total response can lie thought of as the sum of geometrical, penetration, and scatter components.

These components can be defined operationally as mutually exclusive categories of response that function in parallel. These definitions are based on three experiments which may be performed with point sources, but which, for the sake of simplicity, are usually performed with line sources, as depicted in Fig. 3.

a) The geometrical component, $g$, alone is observed by imaging a line source of some low-energy photon emitter in air. Under these conditions, the penetration and scatter components are assumed to be negligible; thus the designation gips for the geometrical component in Figs. 3 and 4 .

b) The geometrical and penetration components are observed together when a line source of the radionuclide to be used clinically is imaged, again in air. The penetration ${ }^{3}$ component can be isolated by subtracting an appropriately normalized ${ }^{4}$ geometrical component obtained in the first experiment.

c) By imaging of the same source as in (b), now in a tissueequivalent medium, the scatter component is added. This component can be isolated by subtracting the sum of geometrical and the penetration curves obtained in measurement (b), which is first multiplied by the attenuation factor $e^{-\mu z_{M}}$, where $z_{M}$ is the path length through the intervening medium.

The importance of having a means of separating these components is that we can decide only on this basis how certain imperfections in the image can best be improved; for example, whether image contrast might best be increased by reducing the collimator field of view (to improve geometrical.

${ }^{3}$ The response observed in experiment (b) includes the response to primary photons scattered through small angles on the collimator.walls, not depicted in Fig. 3. (These photons lose little energy, and therefore may give rise to pulses that lie within the window of the pulse height analyzer.) Thus, scatter in the collimator is operationally defined as septal penetration. In practice, collimator scatter is usually negligible compared with septal penetration [21].

${ }^{4}$ Proper normalization is not easily achieved in practice: (1) The use of absolute calibration methods with both sources is tedious experimentally. (2) Normalization of the two response curves to the same peak amplitude is not entirely satisfactory, since this assumes that the penetration component is zero in both measurements when the source is on the collimator axis. (This assumption is more nearly valid for point than for line sources.) Extrapolation of the tails due to penetration into the geometrical region is based on some arbitrary assumption regarding the shape of the penetration response within this region. (4) Filling all of the collimator holes but one (or a few widely separated holes) with small lead shot or powdered tungsten may reduce the septal penetration fraction to a negligible level, and permit normalization of the geometrical response. 
resolution), by increasing the septal thickness (to reduce penetration), on by raising the lower discriminator on the pulse-height analyzer (to reject more scatter).

The response of a camera-type detector system ${ }^{5}$ can be described quantitatively by

$$
p_{0}\left(r_{0} ; r_{0}\right)\left[\frac{\text { counts at } r_{0}}{s e c-\mathrm{cm}^{2}} / \frac{\text { photon emitted at } r_{0}}{\mathrm{sec}}\right] \text {, }
$$

the response to a unit intensity point source due to the sum of geometrical, penetration; and scatter components; that is,

$$
P_{0}\left(r_{0} ; r_{0}\right)=P_{B}\left(r_{0} ; r_{0}\right)+P_{p}\left(r_{0} ; r_{0}\right)+P_{s}\left(r_{0} ; r_{0}\right) \text {. }
$$

In general, parameters that affect $p_{D}\left(r_{0} ; r_{0}\right)$ include the collimaton 6 and crystal geometry, the energy of emitted photons, $E[\mathrm{MeV}]$, the attenuation coefficient, $\mu(r ; E)$, and the geometry of the surrounding medium, the energy resolution of the detector, $R_{E}$, and the window setting of the pulse-height analyzer, $E_{B}$ and $\Delta E$. By defining $P_{D}\left(r_{D} ; r_{0}\right)$ so that the effects of tissue attenuation and scattering are included in the description of the generalized detector system, we avoid the necessity of introducing the concept of an "equivalent" on "effective". object $[22,23]$.

\subsubsection{Detector sensitivity and resolution for a point source}

This description of the detector system response implies that the probability for a photon emitted at $r_{0}$ to be detected at $r_{0}$ is given by. $P_{0}\left(r_{0} ; r_{0}\right) d r_{D}$ (where $\left.d r_{0} \equiv d x_{0} d y_{D}\right)$, and the probability that it will be detected anywhere is given by the integral over the detector plane, which has area $A_{0}\left[\mathrm{~cm}^{2}\right]$ :

$$
\iint_{A_{D}} P_{D}\left(r_{D} ; r_{0}\right) d r_{D} \equiv \bar{p}_{D}\left(r_{0}\right) A_{D} \equiv D_{0}\left(r_{0}\right)
$$

where $\overline{\mathrm{P}}_{D}$ is the average probability over this area.

Altematively, making use of equation (6), we can also express total response to a point source in terms of the geometrical response and the fractional increases due to penetration and scatter; namely,

$$
D_{0}=\vec{P}_{b} A_{6}\left(I+f_{p}+f_{s}\right) \text {, }
$$

where $A_{0}$ is the area of the detector over which $P_{G}\left(r_{D} ; r_{0}\right) \neq 0$ and $\bar{p}_{0}$ is the

\footnotetext{
${ }^{5}$ For a scanning system [23], the corresponding quantity is $P_{D}\left(r_{0} ; r_{0}\right) d r_{0}\left[\frac{\text { counts at } r_{p} / \text { photons emitted at } r_{0}}{\sec }\right]$.

${ }^{6}$ For converging or diverging collimators, which involve a magnification or minification factor $M$, displacements on the object and detector planes are related by $r_{0}=M r_{0}$. To simplify the notation, we shall assume $M=1$ throughout, including the image plane.
} 
avrage geometrical response over this area. The penetration and scatter fructions ${ }^{7}$ are defined, respectively, by

$$
f_{p}\left(r_{0}\right)=\frac{\iint P_{p}\left(r_{0} ; r_{0}\right) d r_{0}}{\iint p_{0}\left(r_{0} ; r_{0}\right) d r_{0}} \text { and } f_{0}\left(r_{0}\right)=\frac{\iint P_{B}\left(r_{0} ; r_{0}\right) d r_{0}}{\iint P_{0}\left(r_{0} ; r_{0}\right) d r_{0}}
$$

where the integration extends over the entire detector area, $A_{0}$.

For camera systems, $D_{0}, \bar{p}_{0}, \bar{p}_{0}, f_{p}$, and $f_{s}$ are essentialiy constant for all points $r_{0}$ on the $z_{0}$ plane that lie within the useful detector field of view, and approach zero fairly rapidly outside this field. They will be treated in the following as quantities having only two values.

Since $D_{0}$ is the magnitude of the total response to a point source of unit intensity, it can be used as a measure of the sensitivity of the detector system.

On the other hand, the spatial resolution of the detector system is determined by the shape of the response to a point source, which is described by the detector point spread function, $P_{0}^{\prime}\left(r_{0} ; r_{0}\right)$, and defined by

This function is normalized so that

$$
\mathrm{P}_{D}^{\prime}\left(r_{0} ; r_{0}\right) \equiv \frac{p_{D}\left(r_{D} ; r_{0}\right)}{\iint_{A_{0}} P_{D}\left(r_{D} ; r_{0}\right) d r_{D}} .
$$

$$
\int_{-\infty}^{\infty} \int_{0}^{\prime}\left(r_{D} ; r_{0}\right) d r_{0}=1 \text {. }
$$

Therefore,

$$
\mathrm{P}_{0}\left(r_{0} ; r_{0}\right)=\mathrm{D}_{\mathrm{O}} \mathrm{P}_{0}^{\prime}\left(r_{0} ; r_{0}\right)
$$

is the product of two factors that may be regarded as measures of the detector sensitivity and spatial resolution, respectively.

\subsubsection{Detector linearity and stationarity}

If the detector system is linear, ${ }^{8}$ the response to a planar object $o\left(r_{0}\right)$ is given by the superposition of responses to all points of the object: For an observation time of $T[\mathrm{sec}]$, the numerical image $n\left(r_{D}\right)$ at the output of the detector system is given by

7These quantities have been designated $P$ and $S$, respectively, in previous work [22-25]; the symbols $f_{p}$ and $f_{\text {s }}$ are consistent with the notation in reference $[26]$.

${ }^{8} \mathrm{~A}$ linear detector is one for which the total output due to two or more inputs taken together is given by the sum of outputs due to these inputs taken separately. When this is invalid, the superposition principle does not apply, and the analysis of the imaging process is seriously complicated. This is not to be confused with spatial linearity, which implies that straight line elements are imaged as straight lines. 


$$
n\left(r_{0}\right)\left[\frac{\text { counts }}{\mathrm{cm}^{2}}\right] \equiv c\left(r_{0}\right)\left[\frac{\text { counts }}{\mathrm{sec}-\mathrm{cm}^{2}}\right] \times \mathrm{T}[\mathrm{sec}] \equiv \mathrm{T} \int_{\mathrm{A}_{0}} \int_{0} \mathrm{P}_{0}\left(r_{0} ; r_{0}\right) o\left(r_{0}\right) \mathrm{d} r_{0}
$$

The shape of $p_{0}\left(r_{0} ; r_{0}\right)$ tends to vary with distance $z_{0}$, but not with transverse shifts of a point source on planes $\left(\mathrm{x}_{0}, \mathrm{y}_{0} ; \mathrm{z}_{0}\right)$ at a fixed distance $z_{0}$, except near the periphery of the detector field of view. 9 For regions of object space over which $p_{p}\left(r_{0} ; r_{0}\right)$ does not change shape, this quantity is said to be shift-invariant on stationary [such regions are called isoplanatic patches $[17,19]$. In this case, the detector response is a function of $\left(r_{0}-r_{0}\right) \equiv\left(x_{0}-x_{0}, y_{0}-y_{0}\right)$,

$$
P_{0}\left(r_{0} ; r_{0}\right)=P_{0}\left(r_{0}-r_{0}\right)=D_{0} P_{0}^{\prime}\left(r_{0}-r_{0}\right)
$$

and equation (13) can be written as a convolution integral over the object area $A_{0}$ :

$$
n\left(r_{0}\right)=T \int_{A_{0}} \int_{0} O\left(r_{0}\right) P_{0}\left(r_{D}-r_{0}\right) d r_{0}
$$

\subsubsection{Detector sensitivity to a uniform object}

For a large, uniform planar object $\overline{0}\left[\frac{\text { photons }}{\mathrm{sec}-\mathrm{cm}^{2}}\right]$ that covers the detector field of view, the expected count density, $n\left(r_{0}\right)$, is essentially constant over this field and zero outside it. Using equation (14), we obtain

$$
n\left(r_{0}\right)=\operatorname{To}^{-} \int_{-\infty}^{\infty} \int_{D}\left(r_{D}-r_{0}\right) d r_{0}=T_{0}^{-} \int_{A_{0}} \int_{P_{D}}\left(r_{0}-r_{0}\right) d r_{0}=T_{0} D_{0}
$$

The first integral is the total probability that photons emitted on the uniform sheet with unit intensity will contribute to the image at $r_{0}$; the second integral is the total probability that a photon from a point source at $r_{0}$ will contribute to the image somewhere. Therefore, $D_{0}$ can be interpreted either as the count density due to a uniform planar object with unit intensity or as the total response to a point source of unit intensity; in either case, it can be used as a measure of sensitivity.

The total response to a large uniform sheet is

$$
\iint_{D} n\left(r_{D}\right) d r_{D}=\bar{n} A_{D}=T \bar{O} D_{0} A_{D} \equiv T \bar{o} S_{D}[\text { counts }] \text {, }
$$

where

${ }^{9}$ For camera collimators with thick septa (designed for high-energy photons), the shape of $p_{0}\left(r_{0} ; r_{0}\right)$ may change appreciably from point to point on such a plane, especially near the collimator face. Even without a collimator, an improperly tuned scintillation camera may exhibit local "hot spots." In either case, the isoplanatic patches may be quite small. In contrast, scanner detectors are inherently shift-invariant. 


$$
S_{0}\left[\mathrm{~cm}^{2}\right] \equiv D_{0} A_{D}\left[\frac{\text { counts/photons emitted }}{\sec / \frac{\text { sec-om }}{2}}\right] \text {. }
$$

'This is an altermative measure of detector sensitivity that is most frequently used to describe scanning systems. It is especially appropriate for this purpose because the objects imaged in nuclear medicine are large compared to the detector field of view of a scanner and cover this field fairly, uniformly; therefore, the total response at each point can be aitributed to an average object intensity $\overline{0}$, with local variations about this average.

In contrast, such objects are frequently smaller than the useful field of view of a camera system, in which case, only a fraction of the total sensitivity $S_{0}$ is utilized.

Thus, if the useful detector field of view, $A_{D}$, contains the object (i.e., $A_{D} \supset A_{0}$ ), the quantity $\frac{A_{0}}{A_{D}} S_{D} \equiv S_{C D}$ describes the sensitivity of a camera detector to the average object intensity $\overline{0}$ in the sense that Tó $\mathrm{C}_{0}$ is the total response, the mean count density over the numerical image being

$$
\bar{n}_{c}=T_{0} D_{0}=\frac{T_{O} S_{c D}}{A_{0}}=T^{-} \bar{O} \frac{S_{D}(\text { camera })}{A_{D}} \text {. }
$$

For a scanning system covering an area $A_{s} \supset A_{0}$ in the same total observation time $\mathrm{T}[\mathrm{sec}]$, the observation time per unit area is $t\left[\frac{\mathrm{sec}}{\mathrm{cm}^{2}}\right]=\frac{\mathrm{T}}{\mathrm{A}_{\mathbf{s}}}$, and the mean count density accumulated over the object is

$$
\bar{n}_{s}=\frac{\text { Tóso (scanner) }}{A_{s}}
$$

It is clear from equations (19) and (20) that, if the scanned area, $A_{3}$, is the same as the camera detector area, $A_{D}$, then it is useful to compare $S_{D}$ (scanner) with $S_{0}$ (camera) since these quantities determine the mean count densities, $\bar{n}_{s}$ and $\bar{n}_{c}$, that are obtainable in $T[\mathrm{sec}]$. If these areas are not the same, however, it is more meaningful to compare $\frac{S_{0} \text { (scanner) }}{A_{s}}$ with $\frac{S_{D} \text { (camera) }}{A_{D} \text {. }}$

${ }^{A}$ For scanning systems, $S_{0}$ has been formulated explicitly in terms that describe the collimated scintillation detector [23]; that is,

$$
S_{D}\left(z_{0}\right)=G e^{-\mu Z_{M}} n \psi\left[1+f_{P}+f_{B}\left(z_{M}\right)\right] \text {, }
$$

where $G\left[\frac{\text { counts }}{\mathrm{sec}}\right.$ photon emitted $]$ is the geometrical efficiency of the focused collimator in nesponse to a uniform planar object that covers the field of view [in this case, $G$ is independent of distance $z_{0}$ ]; $e^{-\mu z_{M}}$ is the attenuation factor for the intervening medium of thickness $z_{m}=\left(z_{0}-z_{c}\right)$ [where $z_{c}$ is the clearance between collimator and patient]; $f_{p}$ and $f_{s}\left(z_{M}\right)$ are the penetration and scatter fractions, respectively; $n$ is the crystal efficiency for unscattered photons, and $\psi$ is the efficiency of the window setting for counting pulses due to unscattered photons.

Using equation (6), we can describe the sensitivity of a camera system to a large uniform sheet, $\overline{0}$, in similar terms; that is, 


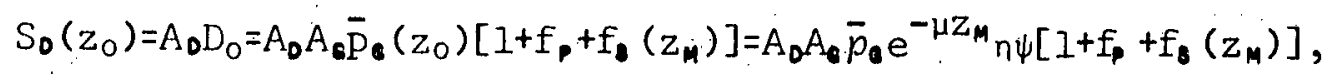

where the factor $\bar{p}_{0}\left(z_{0}\right)$ incorporates the attenuation, crystal, and window effects. Here, $\bar{p}_{\mathrm{l}}$ is the mean geometrical response of the collimator alone, for a point source in air, averaged over $A_{a}$. Thus, for a camera collimator [24],

$$
G=A_{0} A_{c} \bar{p}_{0} \cdot
$$

For a smaller object, with $A_{O} \subset A_{D}$, the effective geometrical efficiency of the collimator is reduced by the factor $A_{0} / A_{0}$. In that case,

$$
G_{0} \equiv \frac{A_{0}}{A_{D}} G=A_{0} A_{0} \bar{p}_{G} \text {. }
$$

2.2.5. Detector Transfer Function $D^{\prime}(v)$ and $\mathrm{MTF}_{0}(v)$

While the mean count density in the image is determined by the magnitude of the detector response (i.e., the detector sensitivity), the shape $^{10}$ of the image is determined by the shape of the detector response to a typical object element (i.e., by the spatial resolution). For a point or line element, the geometrical response tends to be narrow and peaked compared to the penetration and scatter responses. As a result, these components have different effects on the image shape.

The size of the smallest object structures that appear in the image is determined primarily by the narrowness of the geometrical component, which is usually described by the full width at the half-maximum height, FWHM. This quantity can be reganded as a measure of the "resolving power" of the detector system, which is affected very little by the penetration and scatter components. On the other hand, image contrast for all structures of interest may be reduced significantly, relative to object contrast, by the broad, flat penetration and scatter components. Since structure size and contrast concern the shape of the image, a generally applicable measure of spatial resolution would include resolving power and the ability of the system to transfer contrast from the object to the image.

It is appropriate to think of a linear detector system as performing a linear transformation on mapping of object elements onto the image plane; thus, point or line elements are transformed to two- and one-dimensional functions, respectively, which describe the detector response.

For each region over which the detector system is linear and shiftinvariant, a simpler description of this transformation can be found if one inquires, "Does some type of object element exist that is transformed by the detector system in such a way that the image is given by the object element multiplied by a constant?" If so, the detector system could be characterized by a constant multiplier associated with each object element, rather than by a complicated response function.

The answer to this question is yes. The object elements are the sinusoidal plane waves of object intensity, $\odot(v)$, that have been introduced in Fig. I to describe the object. What is important about sinusoids is that, for a linear, shift-invariant system, each object sinusoid is

${ }^{10}$ Images have the same shape if the functions describing the expected count densities differ only by a constant factor. 
transferred to the image as a sinusoid. The efficiency of transfer is described by the constant multiplier, which is the ratio of normalized amplitudes for the image and object sinusoids.

In mathematical physics, the question posed above would be described as an "eigenvalue problem"; the sinusoids are eigenfunctions of the Iinear transformation, and the associated constants are eigenvalues.

To sumarize this in more familiar language, any object and its image can be described in terms of elements that are sinusoidal plane waves. Over each region of object space for which the imaging system is linear and shift-invariant, the object sinusoids will be imaged as sinusoids of the same spatial frequency (except for any magnification factor that may exist), but with reduced amplitude as described by the spatial frequency response of the system. [This is analogous to the (temporal) frequency response of a sound-reproducing system for the (temporal) frequency components of music, etc.]

The assertions above can be expressed formally by starting with equation (15), which describes the expected count density, $n\left(r_{0}\right)$, as a convolution of the object, $o\left(r_{0}\right)$, and the detector system response, $\mathrm{P}_{0}\left(r_{D}-r_{0}\right)$. For three functions related in this manner, the convolutionmultiplication theorem $[17,19]$ states that the Fourier transforms of these functions are related by

$$
N(v)=T \cdot \odot(v) \cdot D(v),
$$

where

$$
N(v) \equiv F \cdot T \cdot\left[n\left(r_{D}\right)\right]
$$

is the unnormalized image spectrum, and

$$
D(v) \equiv F \cdot T \cdot\left[p_{D}\left(r_{0}\right)\right]
$$

is the spatial frequency response of the detector.

For $v=0$, the zero-frequency response or "dc gain" of the detector system is $D(0) \equiv D_{0}$, the detector sensitivity to a uniform object, given by equation (16).

The Detector Transfer Function $D^{\prime}(v)$ is defined by the normalized spatial frequency response of the detector; thus,

$$
D^{\prime}(v) \equiv \frac{D(v)}{D_{0}}=F \cdot T \cdot\left[p_{0}^{\prime}\left(r_{0}\right)\right],
$$

the Fourier transform of the detector point spread function, $p_{0}^{\prime}\left(r_{0}\right)$.

In nuclear medicine, $D^{\prime}(v)$ has heretofore been called the

"Modulation Transfer Function, $\operatorname{MTF}_{D}(\nu)$, of the detector" [27]. In optics, however, and frequently in radiography, the analogous function has been called the Optical Transfer Function, $\operatorname{OTF}(v)$, and its absolute value, $|O T F(v)|$, has been called the Modulation Transfer Function. This dual use of the same term has resulted in some confusion.

In order to facilitate communication between workers in these fields, we suggest that the term Detector Transfer Function be considered for adoption, as the term analogous to Optical Transfer Function, and that the term Modulation Transfer Function be used only to refer to the absolute value, as follows:

$$
\operatorname{MTF}_{0}(v) \equiv \mid \text { Detector Transfer Function }|=| D^{\prime}(v) \mid \text {. }
$$


When defined in this way, $\operatorname{MTF}_{0}(\nu)$ is real and positive, while $D^{\prime}(v)$ may, have positive, negative, or complex values.

If $\mathrm{p}_{0}^{\prime}\left(r_{0}\right)$ is symmetric--this is most frequently a good approximation for the point spread functions encountered in nuclear medicine--then $D^{\prime}(v)$ is a real-valued function that is identical to $\operatorname{MTF}_{D}(\dot{v})$ except when $D^{\prime}(v)$ is negative.

Using equations ( 8 ) and $(28), D^{\prime}(\nu)$ can be expressed in terms of the three components of response; that is,

$$
D^{\prime}(\nu)=\frac{D_{g}^{\prime}(v)+f_{p} D_{p}^{\prime}(v)+f_{g} D_{g}(v)}{1+f_{p}+f_{s}},
$$

where

$$
D_{t, p, s}^{\prime}(v) \equiv \frac{F \cdot T \cdot\left[p_{0, p, g}(r)\right]}{\int_{-\infty}^{\infty} \int_{0, p, s}(r) d r} .
$$

A typical example of these functions is shown in Fig. 5, where the detector can be seen as a low-pass filter. The cutoff frequency, a measure of "resolving power," is determined primarily by the geometrical component. In the mid-frequency range, where $D_{p}^{\prime}(v)$ and $D_{s}^{\prime}(v)=0$, image contrast is reduced by the factor $1 /\left(1+f_{p}+f_{s}\right)$ due to penetration and scatter.

2.3. The numerical image

The numerical image, $n\left(r_{0}\right)\left[\right.$ counts $\left./ \mathrm{cm}^{2}\right]$, is defined by equation (13) for a linear detector, and by equation (15) for a linear, shift-invariant detector. The total expected number of detected photons or "counts" comprising the image is

$$
N_{0}[\text { counts }]=\iint_{A_{D}} n\left(r_{D}\right) d r_{D}=\bar{n} A_{D},
$$

where $\bar{n}\left[\right.$ counts $\left./ \mathrm{cm}^{2}\right]$ is the mean expected count density. This quantity, $N_{0}$, can be used to define the normalized numerical image, $n^{\prime}\left(r_{0}\right)$, and its. spectrum by

$$
n^{\prime}\left(r_{0}\right)=\frac{n\left(r_{0}\right)}{N_{0}} \text { and } N^{\prime}(v)=\frac{N(v)}{N_{0}} \text {. }
$$

With these relations, equation (25) can be put in a form that separates the factors concerning magnitude and shape. To surmarize, the image spectrum

$$
N(v)=T \cdot \odot(v) \cdot D(v)
$$

can be written as the product of two factors,

$$
N_{0} N^{\prime}(v)=T \cdot \odot_{0} \Theta^{\prime}(v) \cdot D_{0} D^{\prime}(\nu),
$$

which can be separated. The first relates counts in the image to the object intensity, detector sensitivity, and observation time, 


$$
N_{0}=T \odot_{0} D_{0}=\bar{n} A_{D}=T \bar{O} A_{O} \bar{P}_{D} A_{D}=T \bar{D} S_{C D},
$$

while the second relates the normalized image spectrum to the normalized object spectrum and the Detector Transfer Function:

$$
N^{\prime}(\nu)=\Theta^{\prime}(\nu) \cdot D^{\prime}(\nu)
$$

2.4. The reconding sytem and the exposure image

The function of the recording system is to store output from the detector for further processing and/or display. Both digital and analog recording systems are currently used. Digital systems typically provide better quantification and pooner subjective image quality than analog systems unless the display matrix consists of $128 \times 128$ elements or more.

Like the detector system, the recording system can be described in terms of its sensitivity and spatial resolution; however, unlike the detector, these characteristics are not ondinarily interdependent in the same way or to the same degree. The photographic recording process will be used to illustrate the general principles involved.

\subsubsection{The Reconder Transfer Function $R^{\prime}(v)$ and $\operatorname{MTF}_{\mathbf{A}}(\nu)$}

Photographic film is the most widely used recording medium. Ordinarily it is exposed by a light source in the form of a spot projector that is pulsed by the detector system output, the exposure distribution 11 for each pulse from the projector, $P_{Q}\left(r_{1} ; r_{0}\right)\left[\frac{\text { light energy at } \mathbf{r}_{1}}{\mathrm{~cm}^{2}} /\right.$ photon detected at $r_{0}$, being equal to the product of light intensity distribution and duration. Ideally, the pulse duration should be as short as possible, consistent with adequate exposure; otherwise, nonlinearity in the relationship between the film exposure and the detector output will occur at high counting rates due to pulse pile-up, and result in reduced contrast. Additional nonlinearity in this relationship will occur unless the exposure per pulse is independent of the count rate ${ }^{12}$ from the detector. This can usually be achieved adequately with cathode ray tubes, and with lightemitting diodes and triodes [28]. .

If such conditions for linearity of the spot projector are satisfied, the expected exposure distribution, $i_{E}\left(r_{1}\right)$, due to the expected numerical image $n\left(r_{0}\right)$ is given by

$$
i_{E}\left(r_{1}\right)=\iint_{A_{D}} n\left(r_{0}\right) P_{R}\left(r_{1} ; r_{D}\right) d r_{D} .
$$

In addition, if the shape of the projected spot does not change over the image area, the projector is shift-invariant and $p_{k}\left(r_{1} ; r_{0}\right)=p_{R}\left(r_{1}-r_{0}\right)$,

${ }^{1}$ This distribution includes the effect of light diffusion within the film; thus, the exposure distribution is slightly larger than the incident light distribution from the projector.

${ }^{12}$ This particular form of nonlinearity is commonly used to increase the image contrast by enabling the exposure per pulse to increase with count rate. 
which reduces equation ( 37 ) for the exposure image to a. convolution:

$$
i_{E}\left(r_{1}\right)=\iint_{A_{D}} n\left(r_{D}\right) p_{R}\left(r_{1}-r_{D}\right) d r_{D}:
$$

When the multiplication theorem for convolution integrals is used, the spatial frequency. spectrum of the exposure image is given by

$$
F \cdot T \cdot\left[i_{E}\left(r_{1}\right)\right] \equiv I_{E}(v)=N(v) \cdot R(v) \text {, }
$$

or

$$
I_{60} I_{E}^{\prime}(v)=N_{0} N^{\prime}(v) \cdot R_{0} R^{\prime}(v)
$$

where

$$
I_{\varepsilon 0} \equiv I_{\varepsilon}(0)=\int_{-\infty}^{\infty} \int_{\varepsilon}\left(r_{1}\right) d r_{1}
$$

is the total exposure, and

$$
R(v) \equiv F \cdot T \cdot\left[p_{R}\left(r_{1}\right)\right]
$$

is the spatial frequency response of the recording systems.

For $v=0$, the zero-frequency response, or "dc gain," of the recording system is equal to the total exposure per. pulse:

$$
R_{0} \equiv R(0)=\int_{A} \int_{n} P_{n}\left(r_{1}\right) d r_{1} \equiv \bar{P}_{n} A_{n},
$$

where $A_{n}$ is the area of the recorded spot and $\vec{p}_{n}$ is the mean exposure over this area. Thus, the Recorder Transfer Function, $R^{\prime}(v)$, defined by

$$
R^{\prime}(v) \equiv \frac{R(v)}{R_{0}} \equiv F \cdot T \cdot\left[P_{n}^{\prime}\left(r_{1}\right)\right]
$$

is the normalized spatial frequency response of the reconder, with $R^{\prime}(0)=1$. $R^{\prime}(v)$ can be thought of as a measure of the spatial resolution of the recording system, and can be computed from the point spread function, $p_{n}^{\prime}\left(r_{1}\right)$. In addition, the modulation transfer function of the recording system is defined by $\operatorname{MTF}_{n}(v) \equiv\left|R^{\prime}(v)\right|$.

From equations (36) and (40), the normalized exposure image spectrum can be expressed as

$$
\begin{aligned}
I_{\varepsilon}^{\prime}(\nu) & =N^{\prime}(\nu) \cdot R^{\prime}(\nu) \\
& =0^{\prime}(\nu) \cdot D^{\prime}(\nu) \cdot R^{\prime}(\nu) \\
& \equiv 0^{\prime}(\nu) \cdot S^{\prime}(\nu),
\end{aligned}
$$

where the System Transfer Function is defined by

$$
S^{\prime}(v) \equiv D^{\prime}(v) \cdot R^{\prime}(v) \text {. }
$$


For $v=0$, equation (40) gives the total exposure to the film,

$$
I_{E O}=N_{0} R_{0}
$$

When the area of the recorded spot, $A_{1}$, is much smallen than the image area, $A_{1}$, so that $A_{0} \cong A_{1}$, the mean density of exposure is given by

$$
\dot{i}_{E}=\frac{I_{E O}}{A_{1}}=\frac{\bar{n} A_{p} R_{0}}{A_{1}}=\bar{n} R_{0}
$$

\subsubsection{Film response}

On development, the film exhibits an image density distribution $i_{0}(r)$ that is, in general; not related linearly to $i_{E}(r)$. Thus, the analysis of the imaging process as a linear transformation is terminated with the exposure image $i_{E}(r)$.

It is important, nevertheless, to examine the relation between exposure and resultant film density since this relationship affects the sensitivity of the recording system:

Film speed or sensitivity, $\mathrm{S}_{\mathrm{p}}$; is typically defined as the reciprocal of the exposure required to produce a certain film density, such as a density of $I_{\text {; thus, }}$ if $i_{R E}$ is the required exposure, equation (48) implies that the film speed is

$$
S_{F}=\frac{1}{i_{n E}}=\frac{1}{n_{n} R_{0}}
$$

where $n_{\mathrm{n}}$ is the required number of counts recorded per unit area, each providing an exposure $R_{0}$, to produce a film density of $l$.

Similarly, the sensitivity of the photographic recording system, $S_{n}$, can then be defined as the reciprocal of the number of counts $/ \mathrm{cm}^{2}$ required to produce a film density of 1 :

$$
S_{n} \equiv \frac{1}{n_{n}}=R_{0} S_{F}
$$

Since there is little difficulty in obtaining intense light sources and sensitive films, a recording system having almost any desired sensitivity can be achieved.

\subsection{The developed image and the observer}

On development of the exposed film, the photographic image may be characterized by its reflection or transmission density, on by some related function such as opacity or transmittance. In general, these functions are not linearly related to the exposure distribution; it is nevertheless appropriate to regand the resultant light intensity distribution associated with the developed image as an input to the visual. system of the observer, and to utilize the methods of linear systems analysis to describe the response of the observer to this input, to the extent that the visual system is linear. For low-contrast images, which present a very limited range of light intensities to the observer, it may be satisfactory to regard the visual system as a linear system $[27,29,30]$. 


\section{PRINCIPLES WHICH GOVERN THE MAXIMUM SENSITIVITY OF THE DETECTOR AND} RECORDING SYSTEMS

Both the detector and recording systems can be described in terms of sensitivity and spatial resolution; however, entirely different principles govern the design of the two systems. In both cases, nevertheless, the design can be carried out in a rational sequence, beginning with the object. The relevant characteristics of the object are the size and contrast of the structures to be detected.

The required spatial resolution of the detector is determined primarily by the size of the smallest object stmucture to be detected in the . image. Various criteria, based on statistical measures of detectability or signal-to-noise ratio, have been proposed for achieving an optimum compromise between detector sensitivity and spatial resolution for a given structure size [27,31-33]. Although these criteria differ in detail, the general principle is the same. The following discussion will be made more concrete by the use of one form of the figure-of-merit criterion, $Q$, to illustrate the principle involved.

Briefly, if $N_{T}=T C_{T}$ and $N_{N}=T C_{N}$ are the expected numbers of counts accumulated in time $\mathrm{T}[\mathrm{sec}]$ over a tumor region (or some other structure to be detected) and a normal region having the same area, 13 respectively, $C_{T}$ and $\mathrm{C}_{\mathrm{N}}$ being the associated count rates, then

$$
T\left(C_{T}-C_{N}\right)=\left(N_{T}-N_{N}\right) \equiv S
$$

is regarded as the signal to be detected. Observed values will be distributed about $\left(\mathrm{N}_{\mathrm{T}}-\mathrm{N}_{\mathrm{N}}\right)$ with a standard deviation

$$
\sqrt{N_{T}+N_{N}} \equiv N \text {, }
$$

which is regarded as the noise accompanying this signal. The signal-tonoise ratio $S / N$ is

$$
S / N=\frac{N_{T}-N_{N}}{\sqrt{N_{T}+N_{N}}}=\frac{\sqrt{T}\left(C_{T}-C_{N}\right)}{\sqrt{C_{T}+C_{N}}} .
$$

The figure of merit; $Q$, is defined by

$$
Q \equiv \frac{(S / N)^{2}}{T}=\frac{\left(N_{T}-N_{N}\right)^{2}}{T\left(N_{T}+N_{N}\right)}=\frac{\left(C_{T}-C_{N}\right)^{2}}{\left(C_{T}+C_{N}\right)}
$$

the value of $(S / N)^{2}$ that can be achieved per unit of observation time. Thus, the quantity

$$
\mathrm{QT}=(S / N)^{2}
$$

can be thought of as an index of confidence in' the detection of a tumor that can be achieved in $\mathrm{T}[\mathrm{sec}]$.

${ }^{13}$ The criterion could be formulated more generally for areas of different size; e.g., the normal region used for comparison may surnound the tumor region. 
The implications of this criterion can be seen more easily when QT is expressed in terms of detector sensitivity and image contrast, which can be defined in several useful ways. The most common measure of image contrast is

$$
\Delta_{2}=\frac{N_{T}-N_{N}}{N_{N}}
$$

which varies from 0 to $\infty$ as $N_{\varphi}$ varies from $N_{N}$ to $\infty$.

Altematively, the quantity

$$
\Delta_{2}=\left(\frac{N_{T}-N_{N}}{N_{T}+N_{N}}\right)
$$

can be reganded as a measure of image contrast that varies from 0 to $I$ as $N_{\mathrm{P}}$ varies from $\mathrm{N}_{\mathrm{N}}$ to $\infty$.

For a low-contrast structure in the object, the image contrast is also low, so that $N_{T} \geq N_{N}$ and $\left(N_{T}-N_{N}\right) \ll N_{N}$. In this case [the most common and difficult one], these measures of image contrast differ by a (nearly) constant factor:

$$
\Delta_{2} \simeq \frac{N_{I}-N_{N}}{2 N_{N}}=\frac{\Delta_{I}}{2}
$$
written

Thus, for the low-contrast case, the detection index, QT, can be

$$
\mathrm{QT}=(\mathrm{S} / \mathrm{N})^{2} \cong \frac{\mathrm{N}_{\mathrm{N}} \Delta_{1}^{2}}{2} \cong 2 \mathrm{~N}_{N} \Delta_{2}^{2} \equiv \mathrm{KN}_{\mathrm{N}} \Delta_{\mathrm{N}}^{2}
$$

where $\mathrm{K}$ is the appropriate constant for the measure of the numerical image contrast, $\Delta_{N}$, that is used. The object contrast; $\Delta_{0}$, can be defined similarly.

\subsection{Application to detector design}

To determine the value of $Q$ for a given object structure and detec-

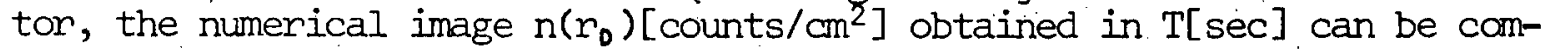
puted from equation (13) or (15) and the value of $N_{T}$ found by integrating $n\left(r_{0}\right)$ over the tumor area $A_{T}$ :

$$
N_{T}[\text { counts }]=\iint_{A_{T}} n\left(r_{D}\right) d r_{D}
$$

Using equation (14) for the detector response function, one can write equation (60) as

$$
N_{T}=\mathrm{TD}_{0} \iint_{A_{T}}\left[\iint_{A_{0}} O\left(r_{0}\right) p_{0}^{\prime}\left(r_{D}-r_{0}\right) d r_{0}\right] d r_{D} ;
$$

similarly, the number of counts over the normal region is 


$$
N_{N}=\operatorname{TD}_{0} \iint_{A_{N}}\left[\iint_{A_{0}} O\left(r_{0}\right) p_{D}^{\prime}\left(r_{D}-r_{0}\right) d r_{0}\right] d r_{D}
$$

Since $N_{1}$ and $N_{N}$ are proportional to $D_{0}$, we can express QT in terms of the detector sensitivity, $D_{0}$, and the numerical image contrast, $\Delta_{N}$ :

$$
\mathrm{QT}=\mathrm{KN}_{\mathrm{N}} \Delta_{\mathrm{N}}^{2}=\mathrm{K}_{\mathrm{N}} \mathrm{D}_{\mathrm{O}} \Delta_{\mathrm{N}}^{2}
$$

where $K_{N}$ is a proportionality factor associated with the normal area $A_{N}$.

Thus, $D_{0}, \Delta_{N}$, and $Q$ can be computed for any object, $o\left(r_{0}\right)$, and any real or hypothetical detector response function, $p_{0}\left(r_{0}\right)$, and in principle, the optimum function which maximizes $Q$, can be found. In general, the optimum response function will depend not only on the tumor size, shape, and contrast, but also on the way in which the tumor area is defined (in object space or in image space), the juxtaposition of the tumor and nomal areas of integration, the form of $p_{0}\left(r_{0}\right)$, and the relation between sensitivity and resolution as $\mathrm{P}_{0}\left(r_{0}\right)$ is altered.

On the basis of most models that have been studied (assuming that the normal area is adjacent to the tumor), the conclusion appears to be that the width of the geometrical component of the detector response function should be made comparable to or slightly larger than the width of the smallest structure to be detected.

Generally speaking, for low-contrast structures, the optimum width is relatively insensitive to variations in object contrast. 'In addition, a significant fraction of the object contrast is transferred to the image by the optimum detector. For a larger field of view, the loss in image contrast is not adequately compensated by the gain in sensitivity, and $Q<Q_{M A x} \equiv Q_{M}$.

These familiar results can be summarized in more general terms:

Detector Principle: The maximum useful sensitivity of the detector system is limited by the size and contrast of the object structures to be detected.

\subsection{Application to recording system design}

Since $N_{N}$ represents the number of counts in a normal region with area $A_{N}$, equal to the tumor area, $A_{T}$, the mean count density is $\bar{n}_{N}=N_{N} / A_{T}$. If we expect this count density to result in a film density of 1 , then equation (50) for the sensitivity of the recording system implies that the sensitivity must be

$$
S_{n}=\frac{I}{\bar{n}_{N}}=\frac{A_{T}}{N_{N}}
$$

Using this relation in equation (59), we obtain

$$
\frac{K A_{T} \Delta_{N}^{2}}{S_{R}}=K \bar{n}_{N} A_{T} \Delta_{N}^{2}=Q T \text {. }
$$

It is important to note that the recorder sensitivity, unlike the detector sensitivity, is inversely proportional to the detection index QT, and if $Q=Q_{M}$ has been maximized by proper detector design, the required value of $S_{n}$ to achieve a film density of 1 over the normal tissue is 


$$
S_{R}=\frac{K A_{T} \Delta_{N}^{2}}{Q_{M} T}
$$

Even if an optimized detector is not used, any required degree of confidence in detection, as measured by $(Q T)_{n}=(S / N)_{R}^{2}$, can be achieved, given sufficient observation time. In principle, this confidence can be achieved with any detector for which $Q \neq 0$.

In general, the mean count density, $\bar{n}_{N h}$, required over the normal region depends on $A_{T}, \Delta_{N}$, and (QT), and can be determined from equation (65). Thus, the required detection index can be achieved on exceeded if $\bar{n}_{N} \geq \bar{n}_{N A}$, and if the reconder sensitivity is reduced accondingly to achieve a film density of 1 :

$$
S_{R} \leq \frac{K A_{T} \Delta_{N}^{2}}{(Q T)_{R}}=\frac{K A_{T} \Delta_{N}^{2}}{(S / N)_{n}^{2}}=\frac{A_{T}}{N_{N R}}=\frac{1}{\overline{n_{N R}}}
$$

If the reconder sensitivity exceeds $I / \bar{n}_{N n}$, a film density of $I$ will be reached before sufficient counts are accumulated to reduce the quantum noise to the required level.

This result can be stated in more general terms: Recorder Principle: The maximum useful sensitivity of the recording system is limited by $\Pi_{N n}$, the count density over normal tissue required to achieve the desired signal-to-noise ratio in the image for the structures to be detected.

\section{IMAGE TMPERFECTIONS, REMEDIES, AND COMMENTS}

A typical image of the distribution of radioactivity within a patient can be thought of as an imperfect projection of a three-dimensional object onto a plane.

\subsection{Image imperfections due to random phenomena}

The inage, as it appears at the detector system output, can be described in terms of the expected count density at each point on the image plane, that is, $n\left(r_{0}\right)$ [counts/cm$\left.{ }^{2}\right]$. Over a finite region with area $A\left[\mathrm{~cm}^{2}\right]$, the expected number of counts is simply

$$
N_{A} \equiv \iint_{A} n\left(r_{0}\right) d r_{0}=\bar{n} A
$$

Due to the random nature of radioactive decay, the number of counts observed within this region on repeated scans would be Poisson-distributed about $N_{A}$ with a standard deviation $\sigma_{A}=\sqrt{N_{A}}$. The fractional standard deviation,

$$
E_{A} \equiv \sqrt{N_{A}} / N_{A}=1 / \sqrt{N_{A}}
$$

is a measure of the relative random error or noise in the determination of $N_{A}$. To the extent that $\varepsilon_{A}>0$, the image quality is limited by random fluctuations or noise. This limitation can be reduced by increasing $\mathrm{N}_{A}$ or $n\left(r_{0}\right)$, which can be achieved by increasing the examination time, the quantity of administered radioactivity, or the sensitivity of the detector system, the last being the most desirable approach. 
While some increase in sensitivity can be achieved through optimization of the collimator design $[22,24]$ using tungsten or gold instead of lead; it appears to be more promising to increase the area and intrinsic efficiency of the detector per se. For conventional scanners, this is best achieved with multiple detectors of moderate diameter, unless the short depth of field associated with lange-diameter detectors is wanted.

Depth of field is not so limited with large-area position-sensitive detectors, which can be used in stationary and hybrid scanning modes to combine high sensitivity with great depth of field $[2,16,34]$.

\subsection{Imperfections due to non-random phenomena}

Even if the object intensity, detector sensitivity, and examination time were essentially unlimited, so that any number of counts could be accumulated to reduce the image noise to a negligible level, the image would nevertheless represent an imperfect projection of the object onto a plane. These imperfections can be described as follows:

4.2.1. Linear distortion due to the detector system: $M_{T F}<1$

To the extent that the system transfer function, $S^{\prime}(\nu)=D^{\prime}(\nu) \cdot R^{\prime}(\nu)$, is less than one for frequency components comprising the object; 14 the image will suffer from linear distortion, which can be seen as smoothing or loss of contrast. The system transfer function can be thought of as a generalized measure of spatial resolution in the sense that it provides a measure of two characteristics that are usually treated separately, namely, resolving power and the ability of the system to transfer contrast from the object to the image. The concept of resolving power is traditionally. defined in terms of the minimum spacing of point on line object elements such that they can be seen as distinct in the image. This corresponds essentially to the high-frequency cutoff point of the system, which is determined primarily by the narrowest component of detector response, i.e., the geometrical component.' While the penetration and scatter responses have little effect on the cutoff frequency on resolving power, they have a significant effect on the image contrast by reducing the magnitude of the transfer function in the mid-frequency range, as shown in Fig. 5.

Linear distortion due to a low cutoff frequency can thus be reduced by narrowing the geometrical field of view of the detector. Increased septal thickness (or the use of tungsten or gold collimators) reduces the loss of contrast due to septal penetration. Increased baseline setting, especially with semiconductor detectors $[25,35]$, , permits the rejection of scattered photons that would reduce image contrast. Generally speaking, these alterations reduce the detector sensitivity and thus $\mathrm{N}_{\mathrm{A}}$, with the result that increased noise is the price paid for reduced linear distortion.

In addition, image contrast may be reduced by motion bluming, due to patient motion in static imaging procedures and motion of the radioactivity in dynamic studies. Correction circuits may be used to reduce the effects of patient motion to some extent [36]; however, increased detector sensitivity to reduce imaging time appears to be the most attractive means of combating motion bluming in dynamic studies.

${ }^{14}$ For a real object, $o\left(r_{0}\right)$, which is non-zero over only a finite region of space, the frequency spectrum $O(\nu)$ extends over all frequency space. 
All of these effects may be described in terms of reduced magnitude of the detector transfer function; that is, $\left|D^{\prime}(v)\right|=\mathrm{MTF}_{0}<1$.

\subsubsection{Linear distortion due to the recording system: $M_{T F} \neq 1$}

The relationship between the Recorder Transfer Function, $R^{\prime}(\nu)$; and the shape of the projected spot, $p:\left(r_{1}\right)$, is indicated in equation (44).

If a very tiny dot is recorded for each pulse from the detector system, then $\left|R^{\prime}(v)\right| \equiv \operatorname{MTF}_{n}(v)=1$ from $v=0$ up to a very high frequency. As the width of the recorded spot is increased, the high-frequency response is reduced. Since there is little restriction on the shape of spots that could be recorded, it is of interest to inquire how the choice should be made.

Apparently nothing objective is gained by having $R^{\prime}(v) \neq 0$ when $D^{\prime}(v)=0$. If the projected spots have the same shape as the detector system response to a point source, then $R^{\prime}(v)=D^{\prime}(v)$. In that case, the system transfer function is $S^{\prime}(v)=\left[D^{\prime}(\nu)\right]^{2}$.

This amount of smoothing is generally regarded as excessive, and it is common practice to record smaller spots, so that $R^{\prime}(\nu) \geqslant 1$ over the frequency range for which $D^{\prime}(\nu) \neq 0$ and decreases fairly rapidly beyond this range. In that case, the overall frequency response is essentially that of the detector alone.

4.2.2.1. A generalization: On-line image processing utilizing a bipolar recording medium

Although it is common practice to smooth the recorded image somewhat by projecting unipolar spots of finite size in the recording system, it is of interest to speculate briefly on the possibility of designing a reconding system with a transfer function $R^{\prime}(v)$ that would perform other imageprocessing functions such as compensating for the mid- and high-frequency attenuation, or linear distortion, introduced by the detector system, so as to sharpen the exposure image or to provide a constant overall response; that is, $D^{\prime}(v) \cdot R^{\prime}(v)=1$. This would be analogous to the use of an equalization filter in sound reproduction to achieve a flat frequency response, as indicated in Fig. 1, except that here the filter would be incorporated "on-line" in the reconding system. Alternatively, we may wish to perform some more general operation; for example, to suppress low and high frequencies and emphasize the mid-frequency range.

The projected-spots that could achieve these functions have a bipolar shape; that is, $p_{n}^{\prime}(r)$ would have a positive central region surrounded by concentric regions which altemate between negative and positive values. To implement this, a recording medium capable of accepting a bipolar input would be required. This simply means that the reconding medium must be capable of repeated operations of local storage and erasure. Photographic film (utilizing the Herschel effect), photochromic materials, and charge storage tubes possess this property [37]. For example, Kelly [38] has shown that, for Kodak Autopositive film, the latent image density can be increased by exposure to blue (short wave length) light and decreased by exposure to red (long wave length) light, and that image sharpening can be achieved by utilizing a blue central spot surrounded by a red annular ring to expose this film (see Fig. 6). It appears that, in principle, many image sharpening functions, including certain non-linear, non-stationary, and adaptive functions, could be performed on-line in the recording system by means of suitable bipolar point spread functions. 


\subsubsection{Effects of inage processing on noise}

In general, the point spread function of the recording system, $p_{h}(r)=\frac{P_{n}(r)}{R_{0}}$, can be thought of as a normalized processing function that operates on the numerical image, $n\left(r_{0}\right)\left[\right.$ counts/cm $\left.{ }^{2}\right]$, to produce the exposure image, $i_{E}\left(r_{1}\right)$. Such processing functions affect the random fluctuations or noise in the processed image in a complex way. An analogous problem occurs in statistical communication theory where a number of authors [19,39-42] have used the autocovariance function as a measure random structure in processed signals. Metz [43] has utilized the autocovariance function to measure the magnitude and character of noise in processed radionuclide images. For zero spatial displacement of the processed image with respect to itself, the autocovariance is equal to the variance, which is the square of the standard deviation. In short, for an input image $n\left(r_{0}\right)$ governed by Poisson counting statistics, and transferred through a linear, shiftinvariant system, the standard deviation associated with the count density at each point in the processed image [in this case, $i_{\varepsilon}\left(r_{1}\right)$ ] is given by

$$
\sigma_{E}\left(r_{1}\right)=\sqrt{\int_{-\infty}^{\infty} \int_{\infty} n\left(r_{0}\right)\left[p !\left(r_{1}-r_{0}\right)\right]^{2} d r_{0}} .
$$

In addition, Metz [43] has shown that the standand deviation associated with unit area in the processed image is given by

$$
\sigma_{E \cup A}\left(r_{1}\right)=\sqrt{\int_{-\infty}^{\infty} n\left(r_{D}\right)\left[h\left(r_{1}-r_{0}\right)\right]^{2} d r_{0}},
$$

where

$$
h\left(r_{1}\right)=\int_{-\infty}^{\infty} \int_{0} u\left(r_{0}\right) p:\left(r_{1}-r_{0}\right) d r_{0},
$$

and $u\left(r_{D}\right)$ is a function having value 1 over the unit area of integration with which $\sigma_{E U_{A}}$ is associated, and zero outside this area. We can think of $\sigma_{E U_{A}}\left(r_{1}\right)$ as a measure of the random structure or noise per unit area in the exposure image $i_{t}\left(r_{1}\right)$. The noise associated with any other area can be defined similarly.

The effect of processing on this measure of noise can be seen most easily by considering the special case in which $n\left(r_{0}\right)=n$ is constant, that is, the expected image of a uniform object. In this case,

$$
\sigma_{E \cup A}=\sqrt{n} \sqrt{\int_{-\infty}^{\infty}[h(r)]^{2} d r} \equiv \sqrt{n} K_{E},
$$

which yields the well known result, $\sigma_{\mathrm{EUA}}=\sqrt{n}$ when $K_{\mathrm{E}}=1$.

From the definition of $u(r)$,

$$
\int_{-\infty}^{\infty}[u(r)]^{2} d r=\iint_{-\infty}^{\infty} u(r) d r \equiv 1 .
$$


Thus, if $p_{h}^{\prime}(r) \geq 0$ for all $r$ and is of finite width, then $h(r) \geq 0$ for all $r$, so that

$$
\int_{-\infty}^{\infty}[h(r)]^{2} d r<\iint_{-\infty}^{\infty}[u(r)]^{2} d r=1, \text { and } K_{E}<1
$$

In this case, the processed image is smoothed in the sense that random structure measured by $\sigma_{\mathrm{tun}}$ is reduced.

On the other hand, if $\mathrm{p} !(r)$ is bipolar, e.g., a typical sharpening function, then it may happen that

$$
K_{E}^{2}=\int_{-\infty}^{\infty} \int_{-\infty}[h(r)]^{2} d r>\int_{-\infty}^{\infty} \int_{\infty}[u(r)]^{2} d r=1 .
$$

In that case, $K_{\varepsilon}>1$, and $\sigma_{\text {EUA }}$ is increased. It is in this sense that random structure or noise may be increased by processing procedures which sharpen the image; that is, increased noise is the price paid for reduced linear distortion.

\subsubsection{Interference}

Interference can be described as reduced detectability or perceptibility of structures relevant to an accurate diagnosis due to the superposition of irrelevant structures in the image. An example is the reduced detectability of lesions in the posterior fossa due to the superposition of the lateral sinuses in the image. In some cases, interference may be reduced by use of a tomographic imaging mode which reduces the depth of field [44]; altematively, the transverse section imaging mode achieves a similar effect [45].

\subsubsection{Attenuated projection}

Due to attenuation of primary photons in overlying tissue, the contribution of these photons to the image decreases exponentially with increasing depth of radioactivity within the patient. The coincidence imaging mode with positron emitters eliminates the dependence of sensitivity on depth and permits the calibration of the imaging system for quantitative measurements of the radioactivity distribution [1].

\subsubsection{Artifacts}

Certain characteristics of the imaging system result in image structures which are unrelated to the object. Examples include line structure due to the scan raster of scanning systems, as well as spot structure and scalloping.

For stationary systems, common artifacts include nonuniformity of sensitivity on display, matrix patterns with multiple detector elements, collimator patterns due to thick septa, etc. Artifacts associated with the collimator or detector can be reduced by moving this assembly (and the recording device) relative to the patient so that each point of the image represents an average response over a large area of the detector $[16,34]$. 


\subsubsection{Nonlinear distortion}

If the output of a system or component is not linearly related to the input, the system or component introduces nonlinear distortion. In that case, an object sinusoid is not imaged as a sinusoid. This may or may not be an undesirable"image imperfection," depending on the effect it has on diagnostic image quality.

For systems employing $\mathrm{NaI}(\mathrm{Tl})$ scintillation detectors, a period of a few microseconds is usually required to detect, analyze, and reject or record each event. As a consequence, pulse pile-up.occurs and the observed counting rate does not increase linearly with the quantity of radioactivity present when the count rate exceeds the order of $10^{4}$ counts/sec. This limits the rate at which statistically significant images can be formed, and is an especially serious limitation in the study of dyanmic processes, where the spatial distribution of radioactivity changes rapidly.

The use of multiple independent detectors and buffered storage reduces this limitation somewhat $[1,34]$. The short-duration light pulses from liquid xenon used as a scintillator may also permit an increase in count rate by more than a factor of ten [48] before comparable nonlinearity due to dead time is observed.

The use of background cutoff circuits to improve the subjective image quality by increasing image contrast results in nonlinearity at low counting rates. A similar effect can be achieved by utilizing the nonlinear response of film, as well as by increasing the exposure per pulse from the detector with increasing count rate.

\section{A GENERALIZATION: OPTIMUM-WEIGHTED MULTICHANNEL IMAGING}

In the foregoing discussion, it has been assumed that a single-channel pulse height analyzer is used to select primarily those photons that pass from the object to the detector without scattering within the patient or collimator. Since all radiation detectors have imperfect energy resolution, however, some scattered radiation will inevitably fall within the window.

The optimum window setting depends on the criterion adopted. With the assumption that scattered photons are like background, contributing nothing to the formation of a structured image, it seems reasonable to set the window so as to minimize the statistical error in measuring the unscattered photons in the presence of a background due to the scatter [25].

An altermative criterion is based on the fact that the response function due to scattered photons from a point or line source is broad, but not precisely flat. In this respect, it differs from the expected response to background radiation. To the extent that scattered photons yield a peaked response, they are capable of contributing a non-zero signal to the formation of a structured image. Therefore, maximizing the signal-to-noise ratio or figure of merit, $Q$, has also been suggested as a criterion for optimizing the window setting $[25,27]$.

By either criterion, the single-channel analyzer accepts or rejects pulses from the detector system; that is, it assigns a weighting factor of 1 or 0 to each pulse from the detector.

In a more general approach, the entire spectrum of pulses (which may include multiple primary primary peaks as well as scatter) may be divided into multiple channels according to amplitude, each channel being allowed to contribute to the image with a weight that may be any real number, positive, zero, or negative. The optimum weight for each channel depends upon the size of the object structures to be detected, the sensitivity, spatial 
resolution, and energy resolution of the detector, as well as the criterion of image quality adopted.

For the most common case of noise-limited images having low-contrast structures near the threshold of detectability, maximum signal-to-noise ratio may be the most appropriate criterion. In that case, if $w_{j} S_{j}$ and $w_{i} N_{j}$ are the weighted signal and noise, respectively, associated with the $j$ th channel, and if it is assumed that the signals from all channels add Iinearly so that $S=\sum_{j} w_{j} S_{j}$, and that the noise adds in quadrature so that $N=\sqrt{\sum w_{j}^{2} N_{j}^{2}}$, then the overall signal-to-noise ratio is given by

$$
S / N=\frac{\sum w_{j} S_{j}}{\sqrt{\sum w_{j}^{2} N_{j}^{2}}}
$$

Since the optimum weighting factors maximize $S / N$, they can be determined by solving $\frac{d(S / N)}{d w_{j}}=0$ for $w_{j}$ (optimum); thus, we find that the optimum weight for each channel is proportional to $S_{j} / N_{j}^{2}$, the ratio of signal/(noise) ${ }^{2}$ for that channel. For example, if $S / N$ is defined by equation (53), then equation (57) indicates that the optimum weighting factor can be thought of as a measure of the image contrast, so that the optimum weighting factor for each channel is simply proportional to the contrast that it provides. For large object structures, optimum weights for scattered and unscattered radiation channels are nearly equal, whereas, for small structures, optimum weights for scattered radiation channels are small compared to weights for unscattered channels.

In any case, when optimum weights are used, the resultant overall figure of merit is the sum of $Q$ values for all channels, and

$$
(S / N)_{\text {MAX }}^{2}=\sum_{j}\left(S_{j} / N_{j}\right)^{2}
$$

This sum exceeds the value obtained with an optimized single-channel analyzer setting. Since the image contrast that can be achieved for small structures by means of scattered photons is relatively low, however, we cannot expect the detectability of such structures to be greatly increased by this approach, even with optimum utilization of all scattered radiation.

At the opposite extreme, when the count density is so great that signal-to-noise ratio may be sacrificed, maximizing some measure of correspondence $[27,47]$ between object and image may be a more appropriate criterion. In general, this implies image sharpening, which may be achieved if negative weights are assigned to certain scatter channels; that is, by using scatter subtraction as opposed to the usual scatter rejection. (In some respects, this procedure is analogous to the well-known photographic technique for image sharpening called "unsharp masking.")

It has been demonstrated that image sharpening can be achieved by scatter subtraction [48]; it will probably turn out to be more satisfactory, however, to allow scatter to augment the signal-to-noise ratio, and to perform image sharpening procedures at a later stage of the imaging process; that is, on-line in the recording system, as suggested in section 4.2.2.1, or off-line with analog on digital techniques.

The concept of optimum-weighted multichannel imaging is sufficiently new so that certain of its features deserve special mention. 
For a nonuniform object, the photon energy spectrum will in general change from point to point; therefore, if optimum weights are fixed for all channels, the shape of the aggregate point spread function will also change from point to point. This particular form of optimum-weighted multichannel imaging is therefore a linear, but nonstationary process. This does not exhaust the possibilities, since one can easily conceive of other nonstationary processes as well as nonlinear and adaptive forms of multichannel imaging utilizing variable weights. It is clear, therefore, that image formation with multiple weighted channels provides greater flexibility and generality than is possible with a single channel. In addition, this method for utilizing all detected radiation can be implemented by on-line and off-line techniques with both scanners and cameras. It should be pointed out, however, that, when any multichannel imaging procedure is to be implemented with a camera system, it is most convenient to utilize a system for which the image size is the same for all photon energies used to form the image. This is an inherent property of multi-element matrix cameras such as the Autofluoroscope [34], as well as the single-crystal scintillation camera designed by Tanaka. [49], using delay-line positioning circuitry.

In addition, if the optimum weighting factors are critically dependent on photon energy, this technique could best be implemented with semiconductor detectors [50-52], which provide excellent energy resolution. For the imaging of radionuclides that emit photons with several different energies, the use of semiconductor detectors combined with the optimumweighted multichannel technique appears especially promising.

\section{SYSTEM EVALUATION AND THE ROC CURVE}

One of the most difficult problems in nuclear medicine is that of evaluating the myriad altemative imaging systems and techniques in terms of the accuracy of physician performance under realistic clinical conditions. It appears, however, that the theory of signal detection [53], which has been adapted by Lusted [54] to the context of medical decision making, provides an especially simple format in which to communicate the results of such studies of performance.

The fundamental problem of diagnosis is that of identifying the patient's, disease (if any exists), on the basis of the evidence that can be obtained at acceptable cost measured in terms of inconvenience, risk, dollars, etc. The solution of this problem is complicated by many factors, including the fact that, in the interest of eliminating excessive cost; the evidence is neither complete nor perfectly precise and accurate. As a consequence, in the simplest model of the diagnostic process, the evidence associated with normal and diseased populations can be described by distributions of signs, symptoms, lab results, etc., which overlap to a degree that corresponds to the quality of the information, as shown in Fig. 7. In the context of image evaluation, these distributions might represent the count rates over normal and tumor tissues in the same patient, as shown in Fig. 8.

Because of the overlap of these distributions, the decision that the patient is normal or diseased may be either true or false. Thus, the decisions of the physician may be classified in four categories:

1) True Positive (TP): the patient has the specified disease, and the physician decides correctly that this is true. 
2) False Positive (FP): the patient is normal, but the physician decides incorrectly that this is false. (Designated $B$ in Fig. 7.)

3) True Negative (TN): the patient is normal and the physician decides correctly that this is true.

4) False Negative (FN): the patient has the specified disease, but the physician decides incorrectly that this is false. (Designated $\alpha$ in Fig. 7.)

The conditional probabilities, $\mathrm{P}$, associated with the four decision categories are not independent. In fact,

$$
\text { and } \begin{aligned}
P(T P)+P(F N) & =1 \\
P(T N)+P(F P) & =1
\end{aligned}
$$

Therefore, a plot of $P(T P)$ vs. $P(F P)$ can be thought of as an implicit plot of $P(T N)$ vs. $P(F N)$. In the theory of signal detection, this plot is described as a Receiver Operating Characteristic (ROC) curve. Examples of such curves are shown in the upper portion of Fig. 8.

In the context of medical diagnosis, the quality of the evidence, and the physician's perception and interpretation of it, together determine an ROC curve. In addition, the physician's judgment about that which he perceives determines the percentage of False Positives he is willing to accept, and thus the point at which he operates on the ROC curve. (This operating point is associated with the position $X_{C}$ along the criterion axis in Fig. 7.)

For the purpose of evaluating imaging systems, it is important to distinguish between these factors of perception and judgment, since our primary goal in improving imaging instruments is to increase the perceptibility of structures relevant to an accurate diagnosis, rather than to alter the physician's judgment. By comparing ROC curves, we can determine when we have succeeded, since the best system-physician combination would produce the ROC curve exhibiting the largest percentage of True Positives for the same percentage of False Positives that is regarded as acceptable.

In diagnostic procedures used in nuclear medicine, the physician is called upon to interpret evidence contained in imperfect images of the distribution of radioactivity within the patient. The effects of these imperfections on the performance of the physician in interpreting an image as normal or abnormal are not known precisely. Thus, it is not possible a priori to say precisely what the expected shape of the distribution functions and the resultant ROC curve would be for a given procedure, characterized by a certain organ to be examined, dose of the radiopharmaceutical, imaging system, examination time, physician to interpret the images, etc. In practice, however, the ROC curves can be determined empirically, and it is this information that must ultimately be used in evaluating imaging procedures.

It is possible, nevertheless, to make rough estimates of the effects of image noise and contrast on the ROC curve based on simple statistical models of threshold detection. These models may be interpreted as describing the expected performance of a computer programmed to integrate counts over suspected tumor and normal regions and to make decisions regarding these counts on the basis of some simple criterion. Two such models are illustrated in Fig. 8.

Model 1. Suppose that the expected count density in the numerical image $n\left(r_{0}\right)$ is essentially uniform over normal tissue and higher over a tumor area, if a tumor exists. The numbers of counts observed over normal and 
tumor regions will be distributed about $N_{N}$ and $N_{T}$ with standard deviations $\sqrt{N_{N}}$ and $\sqrt{N_{T}}$, respectively, as discussed in Section 3 . The expected count nates and associated standard deviations are given by

$$
\begin{aligned}
& C_{N} \pm \sqrt{\frac{C_{N}}{T}}=\frac{N_{N} \pm \sqrt{N_{N}}}{T} \\
& C_{T} \pm \sqrt{\frac{C_{T}}{T}}=\frac{N_{T} \pm \sqrt{N_{T}}}{T} .
\end{aligned}
$$

To illustrate the concepts, these distributions are plotted on the right in Fig. 8 for $C_{N}=100$ coints/T[sec] and $C_{T}=120$ counts $/ T[\mathrm{sec}]$. To interpret the image, one might adopt the criterion that, when the count rate over a given area exceeds a certain level, a tumor is indicated. If this critical level is allowed to vary over all possible values, this criterion gives rise to the ROC curve marked $\mathrm{T}$ in the upper right in Fig. 8.

By extending the observation time to $4 \mathrm{~T}[\mathrm{sec}]$, one can reduce the standard deviation or noise associated with $C_{N}$ and $C_{T}$ by a factor of 2 . The degree of overlap of these distributions is thereby reduced, and this change in procedure might be expected to result in increased confidence of diagnosis. If the physician makes precise use of this, and only this, additional information in making the diagnosis, then the increased confidence in diagnosis can be seen if one compares the ROC curves associated with observation periods $\mathrm{T}[\mathrm{sec}]$ and $4 \mathrm{~T}[\mathrm{sec}]$, plotted in the upper right of Fig. 8. A similar increase in confidence might be expected from increasing the radioactive dose administered to the patient, or increasing the sensitivity of the detector, by a factor of 4 , and keeping the observation time at $\mathrm{T}[\mathrm{sec}]$.

Model 2. If figure of merit, $Q$, is used as a criterion for tumor detection, the expected difference in count rates over the suspected tumor and normal regions $\left(C_{T}-C_{N}\right)$, rather than the count rate over the suspected tumor region alone, is regarded as the signal to be detected. Observed values of this difference will be distributed about the expected value $\left(C_{\mathbf{T}}-C_{N}\right)$, with a standard deviation given by $\sqrt{\frac{C_{I}+C_{N}}{T}}$. When no tumor exists, the observed differences will be distributed about zero with a standard deviation $\sqrt{\frac{2 \mathrm{C}_{N}}{\mathrm{~T}}}$. The distributions of differences associated with the tumor and normal cases are shown in the lower left in Fig. 8, and the associated ROC curves for observation periods of $\mathrm{T}$ [sec] and $4 \mathrm{~T}[\mathrm{sec}]$, in the upper left.

For either model, the improved confidence in the diagnosis that results from the longer period of observation is indicated by the shift in the ROC curve toward the upper left corner of the plot of $P(T P)$ vs. P(FP); that is, toward increased percentage of True Positive diagnoses for a given percentaige of Talse Positives.

In addition, any means by which the difference $\left(C_{T}-C_{N}\right)$, or the contrast $\left(C_{T}-C_{N}\right) / C_{N}$, can be increased would also have the effect of shifting the ROC curve toward the upper left comer of the plot of $P(T P)$ vs. P(FP) which corresponds to ideal performance.

Fon asymmetric distribution functions, the ROC curves associated with different diagnostic procedures may cross each other; this results in a certain ambiguity as to which is the better procedure. One way to resolve this ambiguity has been suggested by Metz [55], who has formulated a measure 
of "information content" which can be computed for each point of operation on the ROC curve; thus, for each ROC curve, a point of operation can be found which maximizes the information content, and different procedures can be compared unambiguously in terms of the maximum information content in the image that they provide.

\section{DISCUSSION AND CONCLUSION}

The analysis outlined in Section 2 is sufficiently general to include two special cases that deserve further discussion.

\subsection{The case of circular symmetry}

If the point spread function of the detector, $p_{0}^{\prime}(r)=p_{0}^{\prime}(x, y)$, is circularly symmetric, then $r=\sqrt{x^{2}+y^{2}}$; that is, $P_{0}^{\prime}$ is a function only of the distance from $(x, y)=(0,0)$. Therefore, $D^{\prime}(v)=D^{\prime}\left(\sqrt{v_{x}^{2}+v_{v}^{2}}\right)$ is also circularly symmetric, and a special form of the two-dimensional. Fourier transform relates these functions. Specifically, $D^{\prime}(\nu)$ can be written as a one-dimensional Fourier-Bessel transform, which is also called the Hankel transform of zero onder $[17,19]$; that is,

$$
D^{\prime}(\nu)=2 \pi \int_{0}^{\infty} r P_{0}^{\prime}(r) J_{0}(2 \pi v r) d r,
$$

where $J_{0}$ is a Bessel function of the first kind and zero order. The inverse of this transform is identical in form:

$$
P_{0}^{\prime}(r)=2 \pi \int_{0}^{\infty} \nu D^{\prime}(\nu) J_{0}(2 \pi v r) d \nu .
$$

\subsection{The more general one-dimensional case in rectangular coordinates}

Since circular symmetry is a special case of the general twodimensional case in which

$$
D^{\prime}\left(v_{x}, v_{y}\right)=\int_{-\infty}^{\infty} \int_{0} P_{0}^{\prime}(x, y) e^{-j 2 \pi\left(v_{x} x+v_{y} y\right)} d x d y,
$$

it follows that, when $p_{0}^{\prime}$ is circularly symmetric; the shape of $D^{\prime}\left(v_{x}, v_{y}\right)$ is the same in all directions and can be described by

$$
\begin{aligned}
D^{\prime}(\nu) & =D^{\prime}\left(\nu_{x}, 0\right)=\int_{-\infty}^{\infty} \int_{0} p_{0}^{\prime}(x, y) e^{-j 2 \pi \nu_{x} x} d x d y \\
& =\int_{-\infty}^{\infty}\left[\int_{-\infty}^{\infty} p_{0}^{\prime}(x, y) d y\right] e^{-j 2 \pi v_{x} x_{1}} d x \\
& \equiv \int_{-\infty}^{\infty} L_{0}^{\prime}(x) e^{-j 2 \pi \nu_{x} x} d x,
\end{aligned}
$$


or

$$
D^{\prime}(v)=F \cdot T \cdot\left[L_{D}^{\prime}(x)\right],
$$

where

$$
L_{0}^{\prime}(x) \equiv \int_{-\infty}^{\infty} P_{0}^{\prime}(x, y) d y
$$

is, by definition, the line spread function of the detector. This function is the normalized detector response to a line source of unit intensity per unit length, $L_{0}(x)$, where

$$
L_{D}(x) \equiv \int_{-\infty}^{\infty} P_{D}(x, y) d y
$$

and

$$
L_{0}^{\prime}(x) \equiv \frac{L_{D}(x)}{D_{0}}=\frac{\int_{-\infty}^{\infty} P_{D}(x, y) d y}{\int_{-\infty}^{\infty} \int_{D}(x, y) d y d x}
$$

Equations (82) through (86) imply that, when $p_{0}^{\prime}(x, y)$ is circularly symmetric, the detector transfer function for all directions can be computed from the unnormalized response to a line sounce parallel to the $y$-axis, $L_{0}(x)$, which can be measured easily [26]; that is,

$$
\begin{aligned}
D^{\prime}\left(\nu_{x}, 0\right)=D^{\prime}(v)= & \frac{F \cdot T \cdot\left[L_{0}(x)\right]}{f_{0}^{\infty}} \cdot \\
& \int_{-\infty} L_{0}(x) d x
\end{aligned}
$$

It is important to note that, even when $p_{j}^{\prime}(x, y)$ is not circularly symmetric, the detector transfer function associated with any particular angular orientation with respect to the $v_{x}$ axis is given by the onedimensional Fourier transform of the normalized response to a line source, measured with the source perpendicular to a line at the same angle with respect to the $x$ axis. It follows that the general, two-dimensional detector transffer function can be computed from the response to a line source, measured at a number of angles, even when circular symmetry does not apply [19].

In addition, for any normalized object $o^{\prime}(x, y)$, the relationship between $o_{L}^{\prime}(x) \equiv \int_{-\infty} o^{\prime}(x, y) d y$ and $n_{L}^{\prime}(x) \equiv \int_{-\infty}^{\infty} n^{\prime}(x, y)$ dy is given by 


$$
n_{L}^{\prime}(x)=\int_{-\infty}^{\infty} O_{L}^{\prime}\left(x_{0}\right) L_{0}^{\prime}\left(x-x_{0}\right) d x_{0} .
$$

The convolution-multiplication theorem imples that

$$
N_{L}^{\prime}\left(\nu_{x}\right)=\odot_{L}^{\prime}\left(\nu_{x}\right) \cdot D^{\prime}\left(\nu_{x}, 0\right) \text {, }
$$

where

$$
N_{L}^{\prime}\left(\nu_{x}\right)=N^{\prime}\left(\nu_{x}, 0\right)
$$

and

$$
\Theta_{L}^{\prime}\left(\nu_{x}\right)=\theta^{\prime}\left(\nu_{x}, 0\right)
$$

A similar relation exists for any angular orientation with respect to the $x$ axis [19]. It short, it appears that the features of the general twodimensional case at any particular angle can be computed from the appropriate one-dimensional case, which may be simpler.

\subsection{Nonlinear and nonstationary imaging systems}

It should be emphasized that the analysis of the imaging process outlined in Section 2 is not entirely general; rather, it is strictly applicable only to imaging systems (and processing functions) that are linear and shift-invariant. The requirements of linearity and shift invariance should be regarded as conveniences in that the analysis of object-image relations is very much simpler for idealized systems assumed to possess these properties. The requirement of these properties is not intended to suggest that the highest degree of diagnostic image quality is thereby assured. On the contrary, certain nonlinear operations, such as background cutoff, and certain nonstationary operations, such as variable averaging and density shifting $[56,57]$, may actually improve diagnostic image quality. Indeed it appears that increased attention should be devoted to the analysis and optimum design of more general nonlinear, and nonstationary systems despite the increased difficulty involved.

\subsection{Effect of the discrete nature of nadioactive decay on the observed image}

In the analysis of the imaging process outlined in Section 2, it was assumed that the function $o(r)$ describing the distribution of radioactivity on a plane is continuous and nonrandom; on this basis, the expected image was computed. The fact that these assumptions are invalid for real distributions of radioactivity suggests that we should expect to find certain differences between the sample, or observed image, and the expected, or mean image. To be specific, randomicity of the radioactive decay process has the effect of introducing fluctuations in observed images, which may be interpreted as noise superimposed on the expected image, as discussed in Section 4.1.

The modifying effect of the discontinuous on discrete nature of the decay process is different. If the image is formed by recording a finite number of discrete events due to the detection of individual photons, even in the absence of randomicity the observed image would differ from the expected image described in Section 2. 
In the computation of the expected image, it is also entirely satisfactory to regard the overall imaging system spread function as the convolution of the detector and recorder spread functions. Alternatively, since the convolution operation is commutative, this expected image is identical to that which would be obtained with an imaging system having the detector and recorder spread functions interchanged. Even in the absence of randomicity, however, the observed images would not be identical if the detector and recorder spread functions were interchanged. Again, the reason can be traced to the discrete nature of the decay process with respect to time, which gives rise to a discrete input to the detector and results in discrete pulses at the detector output. Thus, to account for. the differences between expected and observed images, one must consider the modifying effects of both the random and discrete nature of the object.

\subsection{The function of ROC curves}

Since the ultimate criterion for optimum design of imaging systems must be based upon accuracy of diagnosis, it appears that the use of ROC curves will help us to know when we have succeeded in improving diagnostic image quality, by enabling us to separate the factors regarding the perception of the physician from those regarding his judgment.

\section{ACKNOWLEDGMENT}

The authors are especially grateful to Dr. Charles E. Metz for his contribution to our understanding of image noise, and for several suggestions that increased the clarity of the presentation. This work was supported in part by USPHS Grant No. GM-18940 (Center for Radiologic Image Research).

\section{APPENDIX A: GLOSSARY}

Real space: To simplify the notation, we let $(r)$ symbolize points in real space; thus,

$$
(r) \equiv(\vec{r})=(x, y) \text {, or }(\dot{x}, y, z) \text {, or }(x, y ; z)
$$

and $d r \equiv d r=d x d y$, or dxdydz.

Frequency space: Similarly,

$$
\begin{aligned}
& \quad(v) \equiv(\vec{v})=\left(\nu_{x}, \nu_{v}\right) \\
& \text { and } d v \equiv d v_{x} d \nu_{Y} .
\end{aligned}
$$

Fourier transform of $f(r)$ :

$$
F \cdot T \cdot[f(r)] \equiv F(v)=\iint_{-\infty}^{\infty} f(\vec{r}) e^{-j 2 \pi \vec{v} \cdot \vec{r}} d \vec{r}=\iint_{-\infty}^{\infty} f(x, y) e^{-j 2 \pi\left(\nu_{x} x+v_{p} y\right)} d x d y
$$


Inverse Fourier transform of $F(\nu)$ :

$$
F \cdot T^{-1}[F(v)] \equiv f(r)=\iint_{-\infty}^{\infty} F(\vec{v}) e^{j 2 \pi \vec{v} \cdot \vec{r}} d \vec{v}=\iint_{-\infty}^{\infty} F\left(v_{x}, v_{r}\right) e^{j 2 \pi\left(v_{x} x+v_{r} y\right)} d v_{x} d v_{r}
$$

Since the complex exponential has real and imaginary parts

$\left[e^{-j \alpha}=\cos \alpha-j \sin \alpha\right]$, the spectrum of $f(r)$, namely $F(v)$, is in general

also complex, being the sum of Fourier cosine and sine transforms; that is,

$$
F(v)=F_{c}(v)-j F_{g}(v)
$$

The sinusoidal plane waves represented by this complex spectrum have:

Frequency: $|v|=\sqrt{v_{x}^{2}+v_{y}^{2}}$ in the

Direction: $\theta$ (with respect to the $x$ axis)

$$
\theta=\sin ^{-1} \frac{v_{v}}{\mid \nu_{1}}=\cos ^{-1} \frac{v_{x}}{|\nu|} \text {, }
$$

Amplitude: $|F(v)|=\sqrt{F_{c}^{2}(v)+F_{g}^{2}(v)}$, and

Phase: $\phi[$ with respect to the origin $(r) \equiv(0,0)]$

$$
\phi(\nu)=\sin ^{-1} \frac{F_{s}(\nu)}{|F(\nu)|}=\cos ^{-1} \frac{F_{c}(\nu)}{|F(\nu)|} \text {. }
$$

In general, $f(r)$ may represent the expected value of the object $o(r)$, the numerical image $n(r)$, or the exposure image $i_{E}(r)$; then $F(v)$ represents the comesponding spatial frequency spectrum, $\Theta(v), N(v)$, or $I_{f}(v)$, respectively.

In addition, $f(r)$ may represent the expected response of the detector system, $\mathrm{p}_{0}(r)$, or the recording system, $\mathrm{p}_{n}(r)$, to a unit point input; then $F(v)$ represents the comesponding spatial frequency response, $D(v)$ or $R(v)$, of these systems, respectively.

The total magnitude of $f(r)$ within an area $A\left[\mathrm{~cm}^{2}\right]$ is given by

$$
\iint_{A} f(r) d r=\bar{f} A=F(0) \equiv F_{0} \text {, }
$$

where $\bar{f}$ is the average magnitude over $A$, and $F_{0} \equiv F(v)$ for $v=0$.

This total magnitude, $F_{O}$, serves as a normalization factor in the sense that $f(r) \equiv F_{0} f^{\prime}(r)$ and $F(\nu) \equiv F_{0} F^{\prime}(v)$, where $f^{\prime}(r)$ and $F^{\prime}(v)$ are normalized quantities, with $\iint_{-\infty}^{\infty} f^{\prime}(r) d r=1=F^{\prime}(0)$.

In particular, the normalized spatial frequency response of the detector system, $D^{\prime}(\nu)$, is called the detector transfer function, while

$$
\left|D^{\prime}(v)\right| \equiv \operatorname{MTF}_{D}(v)=\sqrt{\left[D_{c}^{\prime}(v)\right]^{2}+\left[D_{s}^{\prime}(v)\right]^{2}}
$$


is called the modulation transfer function of the detector, and

$$
\phi_{D}(v)=\sin ^{-1} \frac{D_{s}^{\prime}(v)}{\left|D^{\prime}(v)\right|}=\cos ^{-1} \frac{D_{c}^{\prime}(v)}{\left|D^{\prime}(v)\right|}
$$

is called the phase transfer function of the detector. Thus,

$$
D^{\prime}(v)=\operatorname{MTF}_{D}(v) \cdot e^{j \phi_{D}(v)}
$$

The corresponding quantities for the reconding system are similarly defined as shown in Table 1.

\section{REFERENCES}

[1] BROWNELLL, G.L., BURNHAM, C.A., WILENSKY, S., ARONOW, S., KAZEMI, H., STRIEDER, D., "New developments in positron scintigraphy and the application of cyclotron-produced positron emitters," Medical Radioisotope Scintigraphy, IAEA, Vienna 1 (1969) 163.

[2] ANGER, H.O., "Sensitivity and resolution of the scintillation camera," Fundamental Problems in Scanning (GOTTSCHALK, A., BECK, R.N., Eds.), Charles C Thomas Publishers, Springfield, III. (1968) 117 .

[3] ROGERS, W.L., HAN, K.S., JONES, L.W., BEIERWALTES, W.H., Application of a fresnel zone plate to gamma-ray imaging, J. Nucl. Med. 138 (1972) 612 .

[4]. BARREIT, H.H., Fresnel zone plate imaging, J. Nucl. Med. 136 (1972) 382.

[5] ANGER, H.O., McRAE, J., Transmission scintiphotography, J. Nucl. Med. 9 (1968) 267.

[6] KUHL, D.E., HALE, J., EATON, W.L., Transmission scanning a useful adjunct to conventional emission scanning for accurately keying isotope deposition to radiographic anatomy, Radiology 87 (1966) 278 .

[7] CAMERON, J.R., SORENSON, J., Measurement of bone mineral in vivo: an approved method, Science 142 (1963) 230.

[8] CAMERON, J.R., MAZESS, R. B., SORENSON, J.A., Precision and accuracy of bone mineral determination by direct photon absorptiometry, Invest. Radiol. 3 (1968) 141.

[9] HOFFER, P.B., JONES, W.B., CRAWFORD, R.B., BECK, R.N., GOTTSCHALK, A., Fluorescent thyroid scanning: A new method of imaging the thyroid, J. Rad. 90.2 (1968) 342. (Also printed in ACRH \#29, 1968, pp. I-5)

[10] HOFFER, P.B., BERNSTEIN, J., GOTTSCHALK, A., "Clinical results in fluorescent thyroid scanning," Ch. 16, Semiconductor Detectors in the Future of Nuclear Medicine (HOFFER, P.B., BECK, R.N., GOTTSCHALK, A., Eds.), The Society of Nuclear Medicine, New York (1971).

[1]] TINNEY, J.F., "In vivo x-ray fluorescence analysis--concepts and equipment," Ch. 15, Semiconductor Detectors in the Future of Nuclear Medicine (HOFFER, P.B., BECK, R.N., GOTTSCHALK, A., Eds.), Society of Nuclear Medicine, New York (1971).

[12] PATTON, J.A., BRILL, A.B., JOHNSTON, R.E., "Potential use of fluorescent scanning for brain-tumor identification,". Ch. 18, Semiconductor Detectors in the Future of Nuclear Medicine (HOFFER, P.B., BECK, R.N., GOTTSCHALK, A., Eds.), Society of Nuclear Medicine, New York (1971). 
[13] CLARKE, R.L., VAN DYK, G., "Compton-scattered gamma rays in diagnostic radiography," Medical Radioisotope Scintigraphy, IAFA, Vienna 1 (1969) 247.

[14] CASSEN, B., CURTIS, L., REED, C., LIBBY, R., Instrumentation of ${ }^{131}$ I use in medical studies, Nucleonics 127 (1951).

[15] ANGER, H.O., Scintillation camera, Rev. Sci. Instr. 2927 (1958).

[16] HARPER, P.V., GOTTSCHALK, A., CHARLESTON, D.B., YASILIO, N.J., "Area scanning with the Anger camera," Ch. 9, Fundamental Problems in Scanning (GOTTSCHALK, A., BECK, R.N., Eds.), Charles C Thomas Publishers, Springfield, II1. (1968) 145.

[17] GOODMAN, J.W., Introduction to Fourier Optics, McGraw-Hill, New York (1968).

[18] BECK, R.N., HARPER, P.V., CHARLESTON, D.B., YASILLO, N.J., Optical processing of radionuclide images, Biomed. Sci. Instr. 6 (1969) 417.

[19] PAPOULIS, A., Systems and Transforms with Applications in Optics, McGraw-Hill, New Yonk (1968).

[20] MERTZ, L., Transformations in Optics, Wiley, New York (1965).

[21] MATHER, R.L., Gamma-ray collimator penetration and scattering effects, Res. Develop. Tech. Rep. USNRDL-TR-174 NS 081-001 (20 May 1957).

[22] BECK, R.N., A theoretical evaluation of brain scanning systems, J. Nucl. Med. 2 (1961) 314.

[23] BECK, R.N., "The scanning system as a whole: General considerations," Ch. 3, Fundamental Problems in Scanning (GOTTSCHALK, A., BECK, R.N., Eds.), Charles C Thomas Publishers, Springfield, IIl. (1968) 17.

[24] BECK, R.N., "Collimation of garma rays," Ch. 6, Fundamental Problems in Scanjing (GOTTSCHALK, A., BECK, R.N., Eds.), Charles C Thomas Publishers, Springfield, IIl. (1968) 71.

[25] BECK, R.N,, SCHUH, M.W., COHEN, T.D., LEMBARES, N., "Effects of scattered radiation on scintillation detector response," Medical Radioisotope Scintignaphy, IAEA, Vienna I (1969) 595.

[26] MacINTYRE, W.J., FEDORUK, S.O., HARRIS, C.C., KUHL, D.E., MALLARD, J.R., "Sensitivity and resolution in radioisotope imaging," Medical Radioisotope Scintigraphy, IAEA, Vienna I (1969) 391.

[27] BECK, R.N., HARPER, P.V., "Criteria for evaluating radioisotope imaging systems," Ch. 30, Fundamental Problems in Scanning (GOTTSCHALK, A.; BECK, R.N., Eds.), Charles C Thomas Publishers, Springfield, Ill. (1968) 348.

[28] BECK, R.N., CHARLESTON, D.B., EIDELBERG, P., HARPER, P.V., The ACRH brain scanning system, J. Nucl. Med. 8 (1967) 1.

[29]. KELLY, D.H., Visual responses to time-dependent stimuli. I. Amplitude sensitivity measurements, J. Opt. Soc. Amer. 51 (1964) 422.

[30] DePALMA, J.J., LOWRY, E.M., Sine-wave response of the visual system. II. Sine-wave and square-wave contrast sensitivity, J. Opt. Soc. Amer., 52.3 (1962) 328.

[31] NEWELL, R.R., SAUNDERS; W., MILIER, E., Multichannel collimators for gamma ray scanning with scintillation counters, Nucleonics 107 (1952) 36.

[32] DEWEY, W.C., SINCLAIR, W.K., Criteria for evaluating collimators used in in vivo distribution studies with radioisotopes, Int. J. Appl. Radiat., 10 I (1961).

[33] MALLARD, J.R., WILKS, R.J., CORFIELD, J.R., FLOOK, V., "Visualization in scanning," Medical Radioisotope Scintigraphy, IAEA, Vienna I (1969), 305.

[34] JONES, R.H., GRENIER, R.P., SABISTON, D.C., Jr., Description of a new high count rate gamma camera system, In these PROCEEDINGS. 
[35] BECK, R.N., ZIMMER, L.T., CHARLESTON, D.B., HOFFER, P.B., LEMBARES, N., "The theoretical advantages of eliminating scatter in imaging systems," Ch. 7, Semiconductor Detectors in the Future of Nuclear Medicine (HOFFER, P.B., BECK, R.N., GOTTSCHALK, A., Eds.), The Society of Nuclear Medicine, New York (1971).

[36] HOFFER, P.B., OPPENHEIM, B. E., YASILLO; N.J., STERLING, M.L., Motion comection in liver scanning: Description of a new device and preliminary clinical results, J. Nucl. Med. 126 (1972) 437. (Abstract)

[37] BECK, R.N., CHARLESTON, D.B., ZIMMER, L.T., BECKMAN, F.H. , Analog methods of data storage, Proceedings of the First Biennial Conference on Quantitative Organ Visualization in Nuclear Medicine, Miami, May 1970 (KENNY, P.J., SMITH; E.M., Eds.), University of Miami Press (1971) 313.

[38] KELLY, D.H., Image-processing experiment, J. Opt. Soc. Amer. 5] (1961) 1095.

(39] LEE, Y.W., Statistical Theory of Communication, Wiley, New York (I960) 332.

[40] DAVENPORT, W.B., ROOT, W.L., An Introduction to the Theory of Random Signals and Noise, McGraw-Hill, New York (1958) 182.

[41] PAPOULIS, A., The Fourier Integral and Its Applications, McGraw-Hill, New York (1962) 246.

[42] THOMAS, J.B., An Introduction to Statistical Communication Theory, Wiley, New York (1969) 144.

[43] METZ, C.E., A mathematical investigation of radioisotope scan image processing, Ph.D. Thesis, University of Pennsylvania, 1969, available from University Microfilms, Ann Arbor, Michigan, Publication \#70-16-168.

[44] ANGER, H.0., "Multiplane tomognaphic garma-ray scanner," Medical Radioisotope Scintigraphy, IAEA, Vienna I (1969) 203.

[45] KUHL, D.E., EDWARDS, R.Q., "Digital procéssing for modifying and rearranging rectilinear and section scan data under direct observation," Medical Radioisotope Scintigraphy, IAEA, Vienna I (1969) 703.

[46] LAVOIE, L., Positron detection system using liqüid xenon scintillator, J. Nucl. med. 126 (1971) 376. (Abstract)

[47] LINFOOT, E.H., Transmission factors and optical design, J. Opt. Soc. Amer. 469 (1956) 740.

[48] BECK, R.N., ZIMMER, L.T., CHARLESTON, D.B., HOFFER, P.B., Aspects of inaging and counting in nuclear medicine using scintillation and semiconductor detectors, IEEE Trans. Nucl. Sci. NS-18 3 (1972).

[49] TANAKA, E., NOHARA, N., KUMANO, N., KAKEGAWA, M., A large area, high resolution scintillation camera based on delay line time conversion, In these PROCEEDINGS.

[50] PARKER, R.P., GUNNERSEN, E.M., WANKLING, J.L., ELLIS, R., "A semiconductor gamma camera with quantitative output," Medical Radioisotope Scintigraphy, IAEA, Vienna 1 (1969) 71.

[51] DETKO, J.F., A prototype, ultra puré germanium, orthogonal strip gamma camera, In these PROCEEDINGS.

[52] PARKER, R.P., GUNNERSEN, E.M., ELIIS, R., BELL, J., A semiconductor $\gamma$-camera: assessment of results, In these PROCEEDINGS.

[53] GREEN, D.M., SWETS, J.A., Signal Detection Theory and Psychophysics, Wiley, New York, (1966).

[54] LUSTED, L.B., Introduction to Medical Decision Making, Charles C Thomas Publisher, Springfield, IIl. (1968). 
[55] METZ, C.E., Enpirical evaluation of imaging procedures in terms of information content and receiver operating characteristic curves, J. Nucl. Med: 13 (1972) 453.

[56] WILENSKY, S., ASHARE, A.B., PIZER, S.M., HOOP, B., BROWNELL, G.L., "Computer processing and display of positron scintigrams and dynamic function curves," Medical Radioisotopes Scintigraphy, IAEA, Vienna I' (1969) 815

[57] MTZ, C.E., PIZER, S.M., "Nonstationary and nonlinear scintigram processing," Proc. Conf. Data Handling and Image Processing in Scintigraphy (TODD-POKROPEK, JAHNS, E., Eds.), Hanover, Germany, October (1971). In press. 


\begin{tabular}{|c|c|c|c|c|c|c|c|}
\hline DESCRIPTION & $\begin{array}{l}\text { GENERAL } \\
\text { RELATION }\end{array}$ & OBJECT & DETECTOR & $\begin{array}{c}\text { NUMERICAL } \\
\text { IMAGE }\end{array}$ & RECORDER & $\underset{\text { IMAGE }}{\text { EXPOSURE }}$ & $\begin{array}{c}\text { DEV, } \\
\text { IMAGE }\end{array}$ \\
\hline $\begin{array}{c}\text { Unnormalized } \\
\text { Spatial } \\
\text { Distribution } \\
\text { or Response } \\
\text { F.T. }+\uparrow F . T .^{-1} \\
\text { Unnormalized } \\
\text { Spatial Frequency } \\
\text { Spectrum } \\
\text { or Response }\end{array}$ & $\begin{array}{c}f(r) \\
+\uparrow \\
F(v)\end{array}$ & $\begin{array}{l}o(r) \\
\downarrow \uparrow \\
O(v)\end{array}$ & $\begin{array}{c}p_{0}(r) \\
t \uparrow \\
D(v)\end{array}$ & $\begin{array}{c}n(r) \\
\ddots \downarrow \uparrow \\
\vdots \\
N(\nu)\end{array}$ & $\begin{array}{c}P_{n}(r) \\
\downarrow \uparrow \\
R(v)\end{array}$ & $\begin{array}{c}i_{\varepsilon}(r) \\
\downarrow \uparrow \\
I_{\varepsilon}(\nu)\end{array}$ & $i_{0}(r)$ \\
\hline $\begin{array}{l}\text { Normalization Factor: } \\
\text { Average X Area } \\
=\text { Total }\end{array}$ & $\begin{array}{c}\bar{f} A \\
=F(0) \equiv F_{0} \\
\end{array}$ & $\begin{array}{c}\sigma A \\
=0(0) \equiv \sigma_{0}\end{array}$ & $\begin{array}{c}\overline{\mathrm{P}}_{0} \mathrm{~A}_{0} \\
=\mathrm{D}(0) \equiv \mathrm{D}_{0}\end{array}$ & $\begin{array}{c}\bar{n} A_{0} \\
=N(0) \doteq N_{0}\end{array}$ & $\begin{array}{c}\overline{\mathrm{P}}_{\mathrm{R}} \mathrm{A}_{\mathrm{R}} \\
=\mathrm{R}(0) \equiv \mathrm{R}_{0}\end{array}$ & $\begin{aligned} & I_{E} A_{1} \\
= & I_{E}(0) \equiv I_{E 0}\end{aligned}$ & \\
\hline $\begin{array}{c}\text { Normalized } \\
\text { Spatial } \\
\text { Distribution } \\
\text { or Response } \\
\text { F.T. }+\uparrow F .^{-1} \\
\text { Normalized } \\
\text { Spatial Frequency } \\
\text { Spectrum } \\
\text { or } \\
\text { Transfer Function }\end{array}$ & $\begin{array}{c}\quad f^{\prime}(r) \\
=\frac{f(r)}{F_{0}} \\
+\uparrow \\
F^{\prime}(v) \\
=\frac{F(v)}{F_{0}} \\
=F_{c}^{\prime}(v)-j F_{s}^{\prime}(v)\end{array}$ & $\begin{aligned} & O^{\prime}(r) \\
= & \frac{\partial(r)}{\sigma_{0}} \\
& +\uparrow \\
& \sigma^{\prime}(v) \\
= & \frac{\partial(v)}{\sigma_{0}} .\end{aligned}$ & $\begin{aligned} & P_{0}^{\prime}(r) \\
= & \frac{P_{p}(r)}{D_{0}} \\
& +1 \\
& D^{\prime}(v) \\
= & \frac{D^{\prime}(v)}{D_{0}} \\
= & D_{c}^{\prime}(v)-j D_{j}^{j}(v)\end{aligned}$ & $\begin{array}{c}\quad n^{\prime}(r) \\
=\frac{n(r)}{N_{0}} \\
+\uparrow \\
N^{\prime}(v) \\
=\frac{N(v)}{N_{0}}\end{array}$ & $\begin{aligned} & P_{R}^{\prime}(r) \\
= & \frac{P_{R}(r)}{R_{0}} \\
& +\uparrow \\
& R^{\prime}(v) \\
= & \frac{R(v)}{R_{0}} \\
= & R_{d}^{\prime}(v)-j R \mathbf{j}(v)\end{aligned}$ & $\begin{aligned} & I_{\varepsilon}^{\prime}(r) \\
= & \frac{I_{\varepsilon}(r)}{I_{\varepsilon 0}} \\
& \downarrow \uparrow \\
& I_{\varepsilon}^{\prime}(\nu) \\
= & \frac{I_{\varepsilon}(\nu)}{I_{E c}}\end{aligned}$ & \\
\hline $\begin{array}{l}\text { Modulation } \\
\text { Transfer Function }\end{array}$ & $\begin{array}{l}\operatorname{MTF}_{F}(\nu) \\
=\left|F^{\prime}(v)\right|\end{array}$ & & $\begin{array}{l}\operatorname{MTF}_{0}(v) \\
=\left|D^{\prime}(v)\right|\end{array}$ & & $\begin{array}{l}\operatorname{MTF}_{n}(v) \\
=\left|R^{\prime}(v)\right|\end{array}$ & & \\
\hline $\begin{array}{l}\text { Phase } \\
\text { Transfer Function }\end{array}$ & $\begin{array}{c}\phi_{F}(v) \\
=\cos ^{-1} \frac{F_{L}^{\prime}(v)}{|F(v)|}\end{array}$ & & $\begin{array}{c}\phi_{D}(\nu) \\
=\cos ^{-1} \frac{D_{C}^{\prime}(v)}{\left|D^{\prime}(v)\right|}\end{array}$ & & $\begin{array}{c}\phi_{R}(v) \\
=\cos ^{-1} \frac{R t(v)}{\left|R^{\prime}(v)\right|}\end{array}$ & & \\
\hline
\end{tabular}




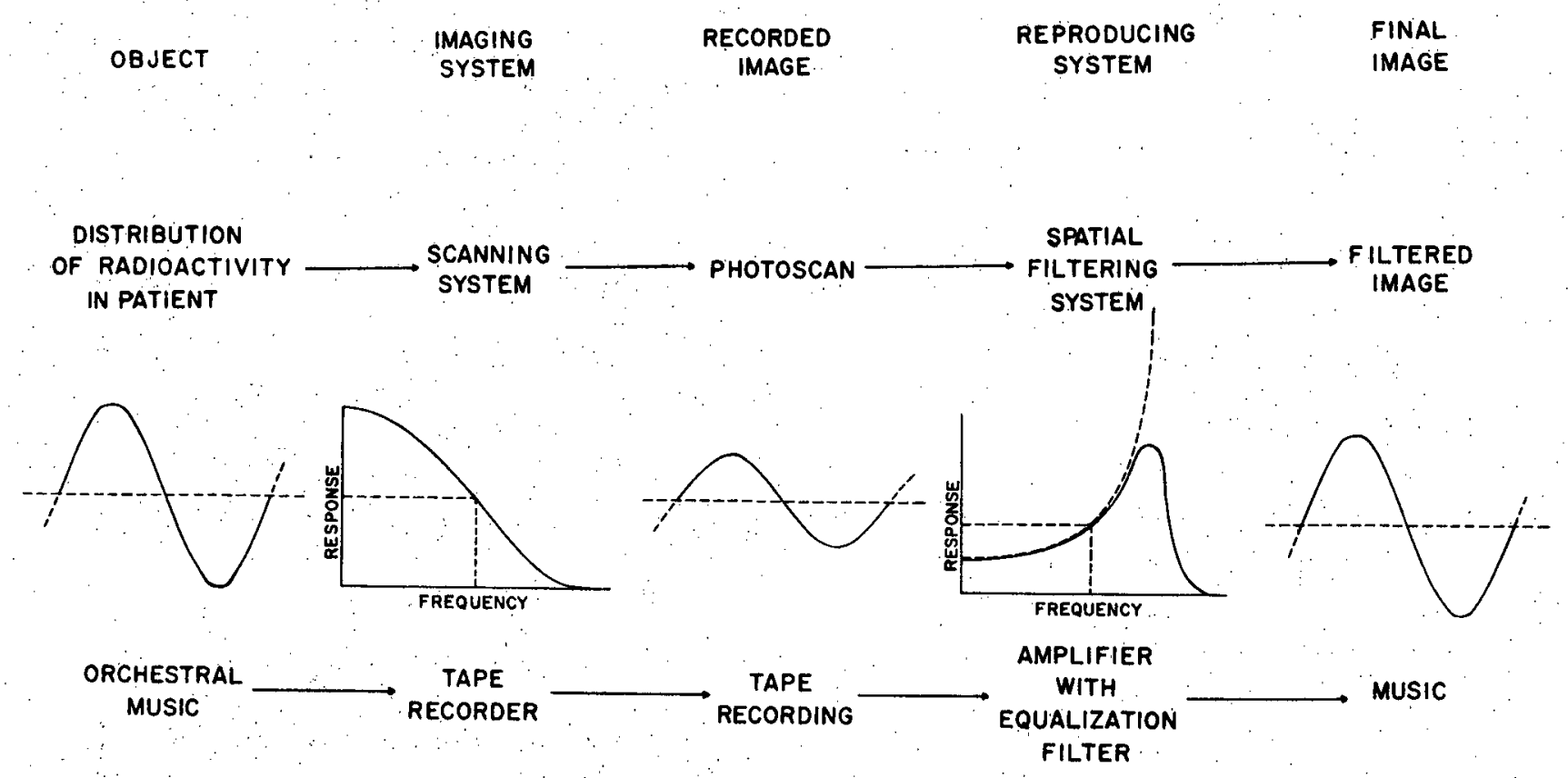

Fig. 1. To the extent that the imaging process is linear and stationary, the analogous concepts and methods used to analyze sound reproducing systems are applicable. 


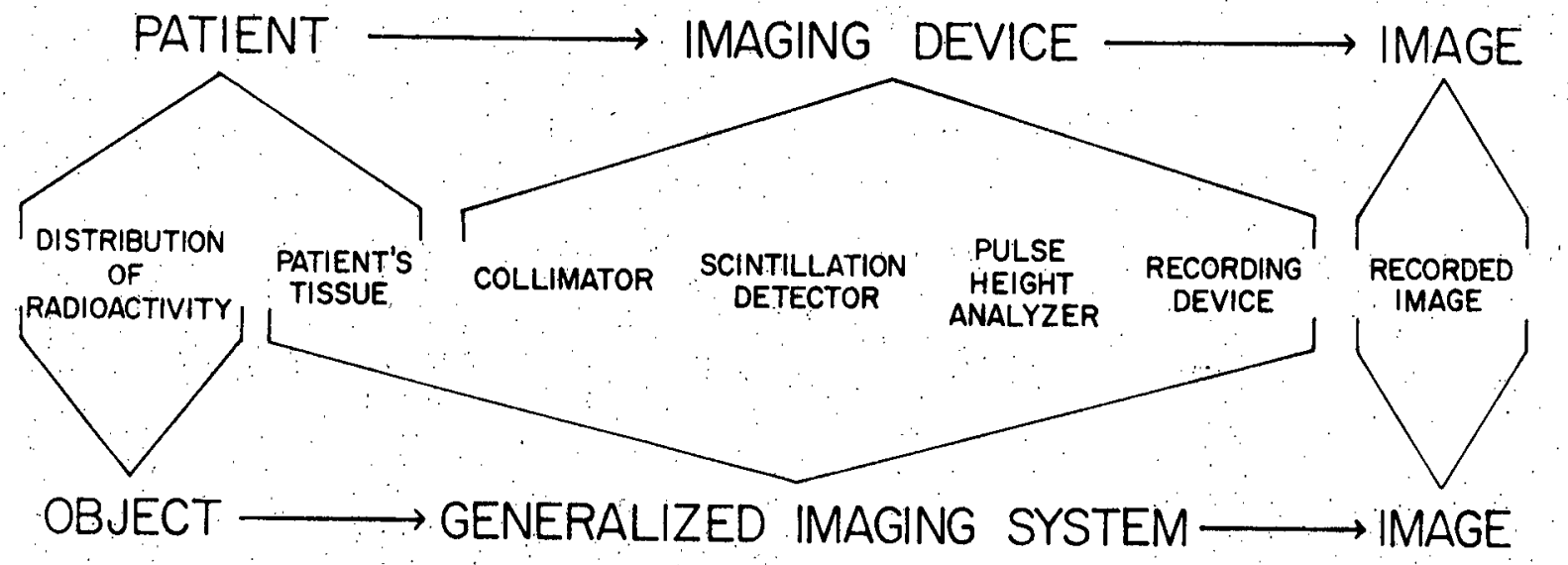

Fig. 2. Everything that intervenes between the object and image is regarded as part of a generalized imaging system. This includes the patient's tissue, which scatters and absorbs radiation from the object. 


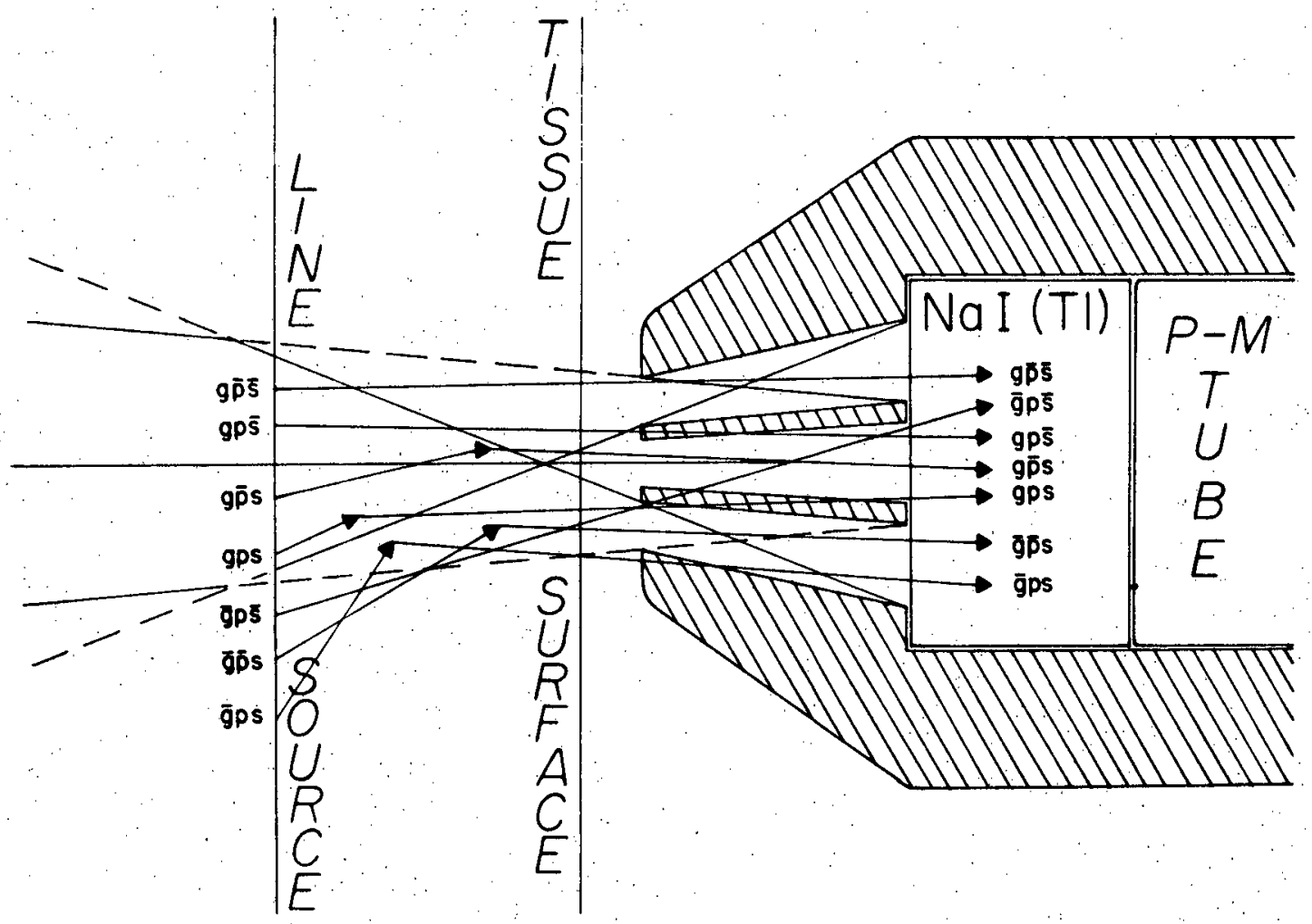

Fig. 3. Photons emitted within the geometrical field of view are designated $g$, and photons not emitted within this region are designated $\bar{g}$; similarly for photons which do $(p, s)$ and do not $(\underline{p}, \bar{s})$ penetrate the collimator septa or scatter within the patient. Thus, gps refers only to those photons which enter the detector properly. 


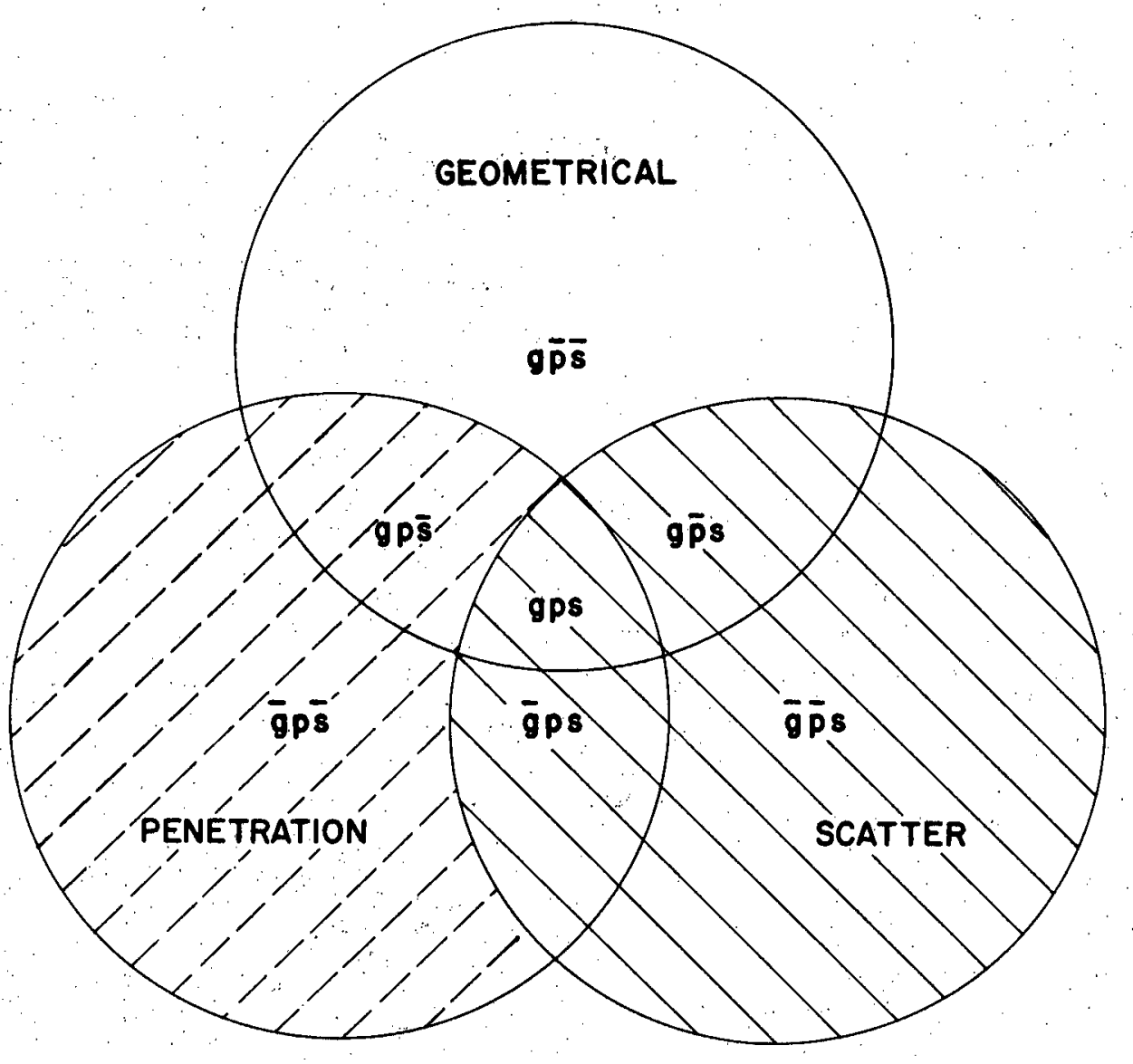

Fig. 4. Events which characterize the detector response can be separated into three mutually exclusive categories by means of three simple experiments described in the text. 


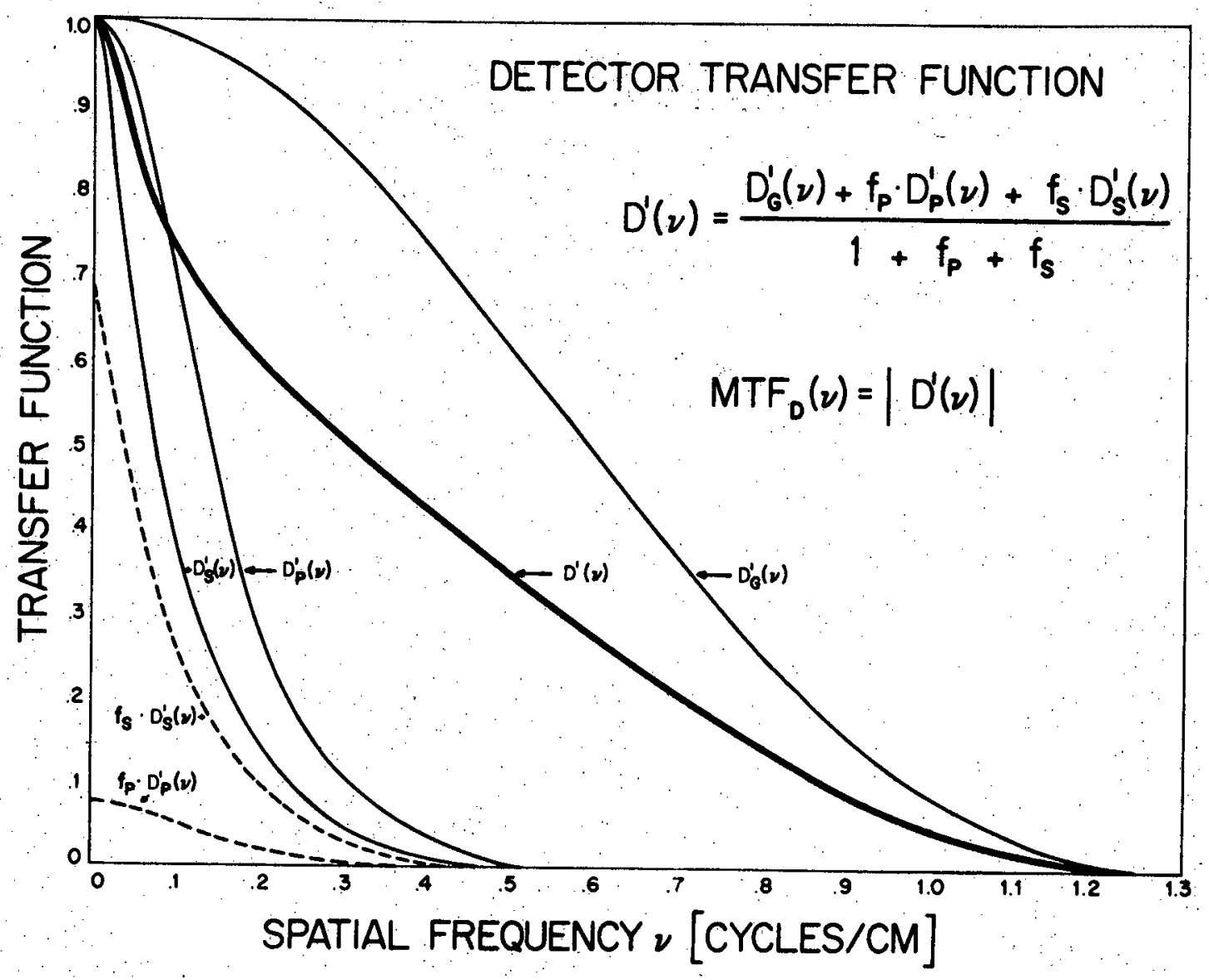

Fig. 5. The Detector Transfer Function $D^{\prime}(\nu)$ is in general a complex-valued function defined by the Fourien transform of the detector point or line spread function. The detector Modulation Transfer Function $\operatorname{MTF}_{0}(v)$ is defined by the magnitude of $D^{\prime}(v)$. Penetration and scatter have little effect on the high-frequency cutoff point, but degrade image contrast by reducing the mid-frequency response. 


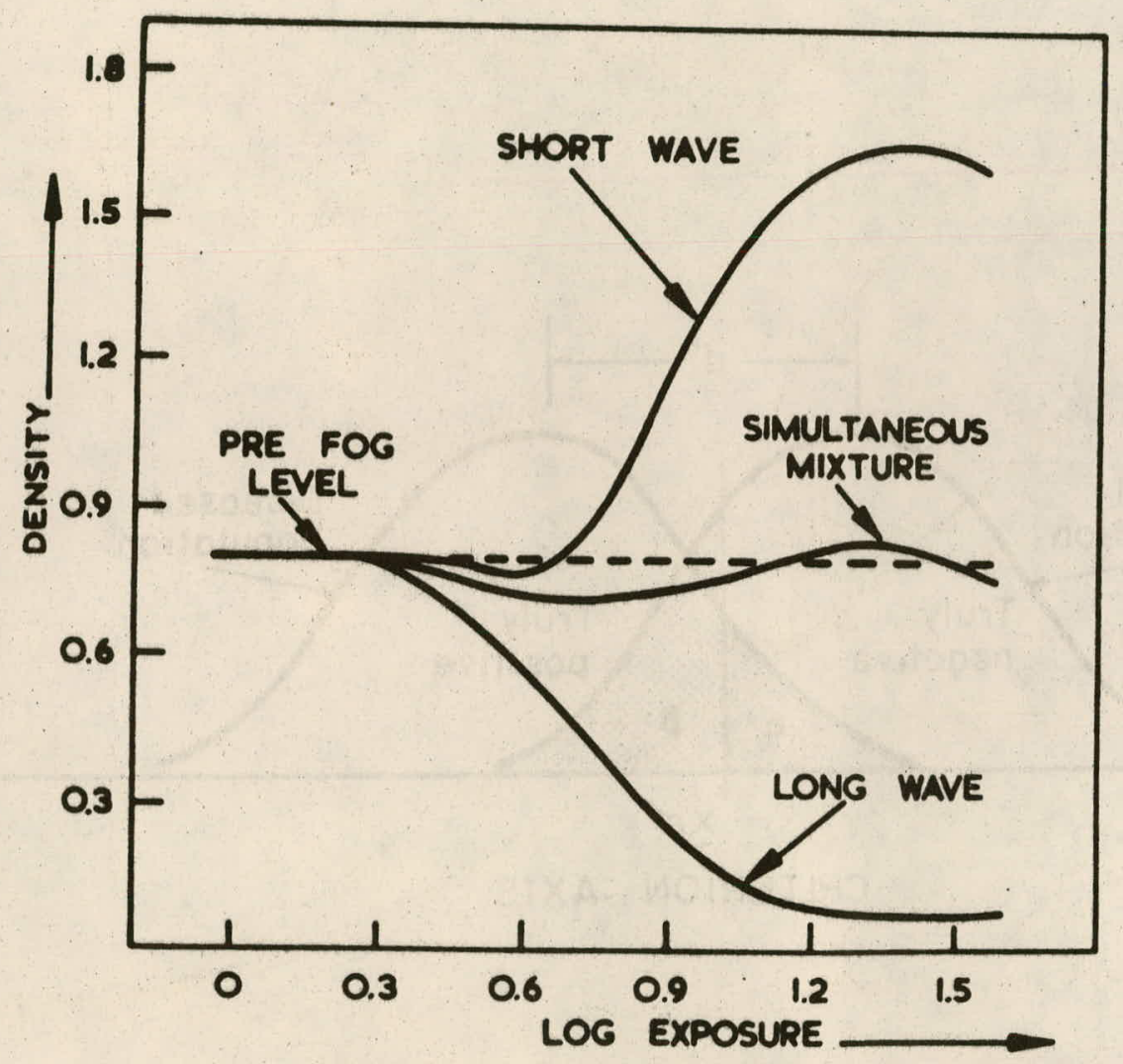

Fig: 6. Kodak Autopositive Film may be reganded as a bipolar reconding medium since the latent film density can be increased by exposure to blue (short wave length) light and decreased by exposure to red (long wave length) light. By projecting a bipolar spot (blue in the center and red in an annular region), one could achieve on-line image sharpening in the recording device. [Reproduced from Ref. 38 with permission of the Journal of the Optical Society of America.] 


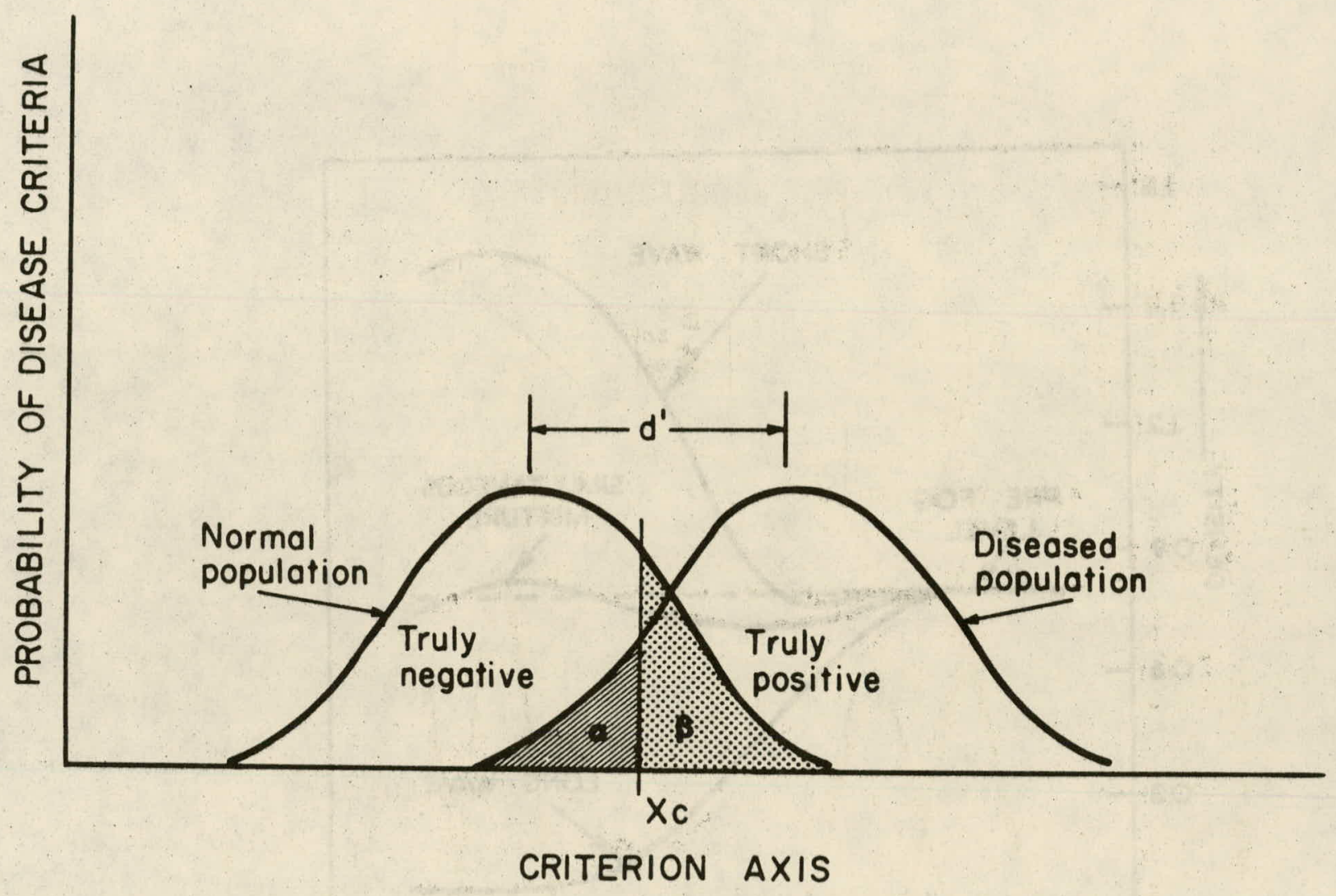

Fig. 7. The quality of the information used by the physician in diagnosis (and his perception of it) determines the apparent degree of overlap of the distributions of signs and symptoms for normal and diseased populations. The physician's judgment regarding acceptable levels of False Positive and False Negative diagnoses (designated $\beta$ and $\alpha$, respectively) determines the critical level, Xc. For the special case of symmetric distributions with the same variance, the number of standard deviations separating the mean values, d', provides an adequate index for comparing diagnostic procedures. In the more general case of asymmetric distributions, the associated ROC curves for different procedures may cross each other. [Reproduced from Ref. 54 with permission of the author.] 

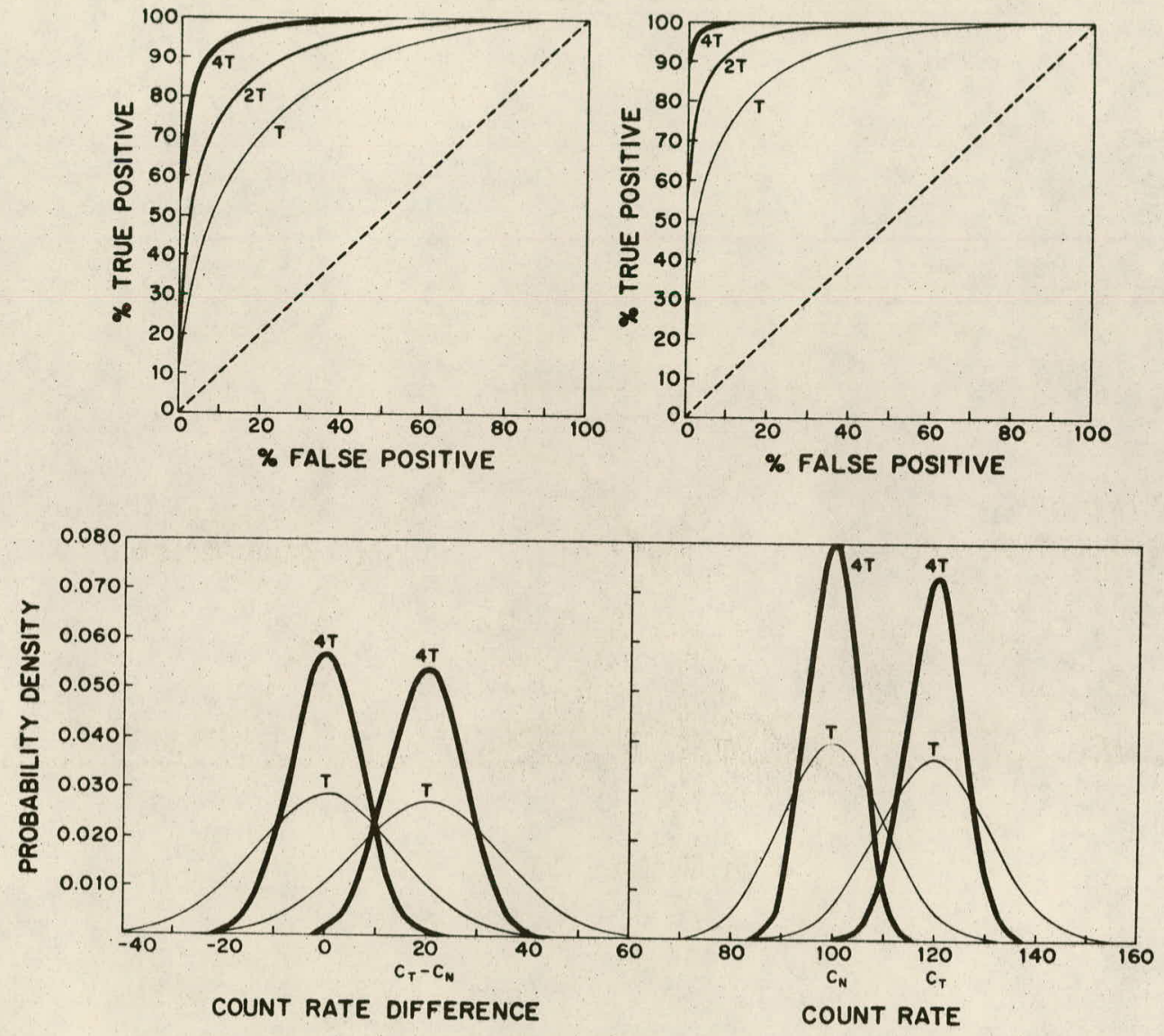

Fig. 8. If count rates over normal and tumor tissues, $C_{N}$ and $C_{T}$, respectively, constitute the diagnostic information, the degree of overlap can be reduced by extending the observation time from T[sec] to 4T[sec]. This increases the accuracy of diagnosis, as indicated by the plot of \% TP vs. $\% \mathrm{FP} \equiv[\%$ Positives Scored Positive vs. $\%$ Negatives Scored Positive], which is called an ROC curve. 


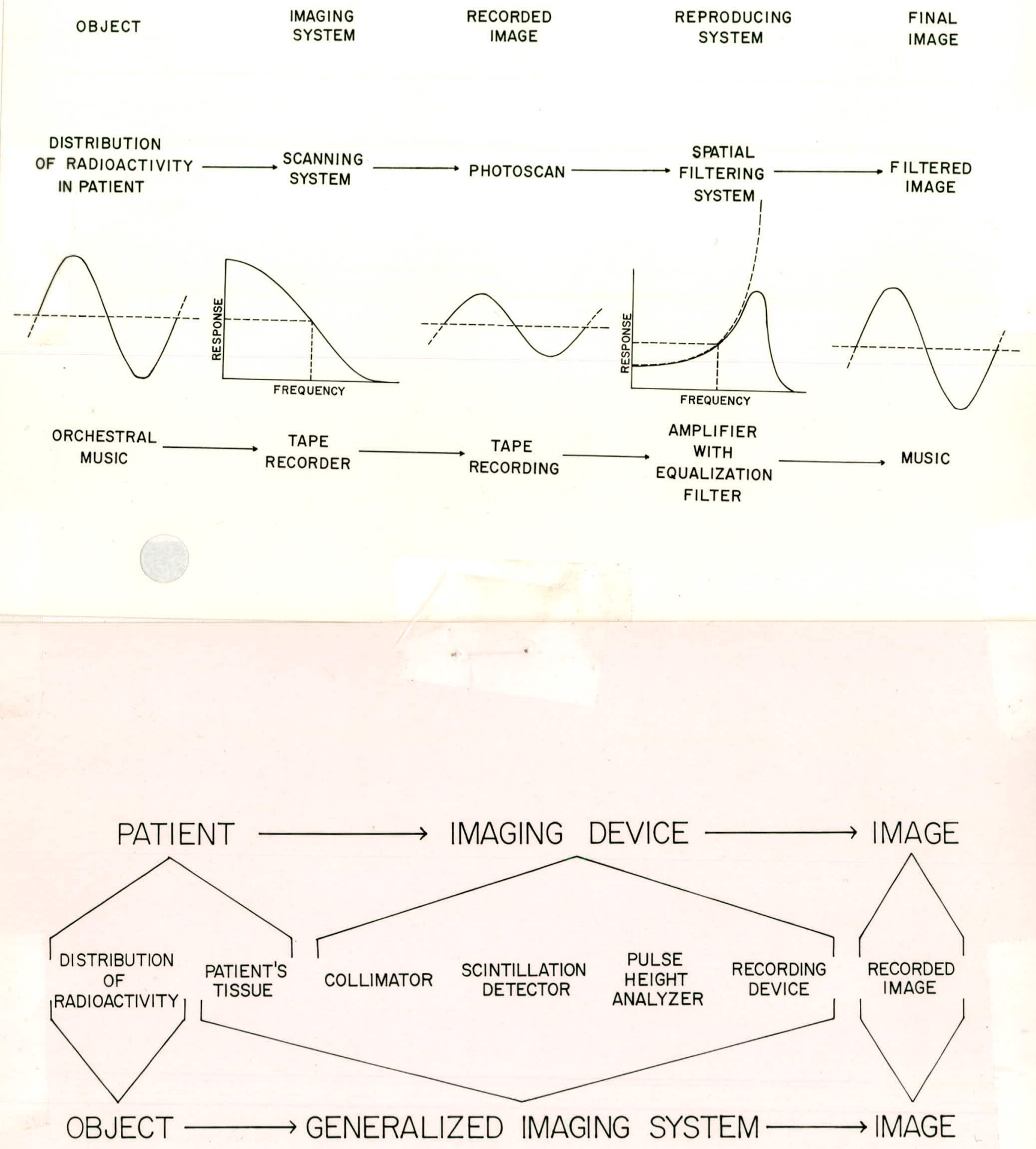



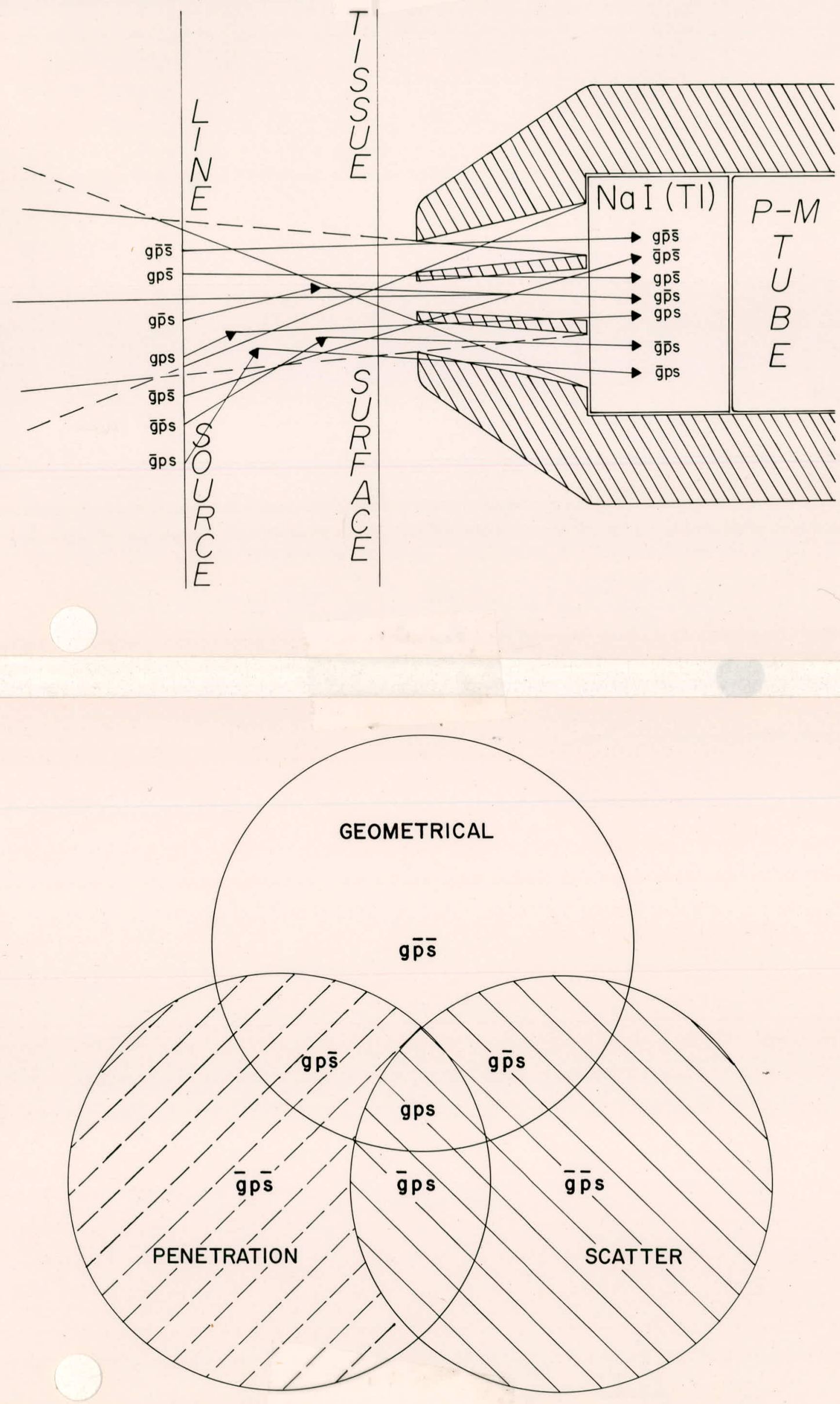

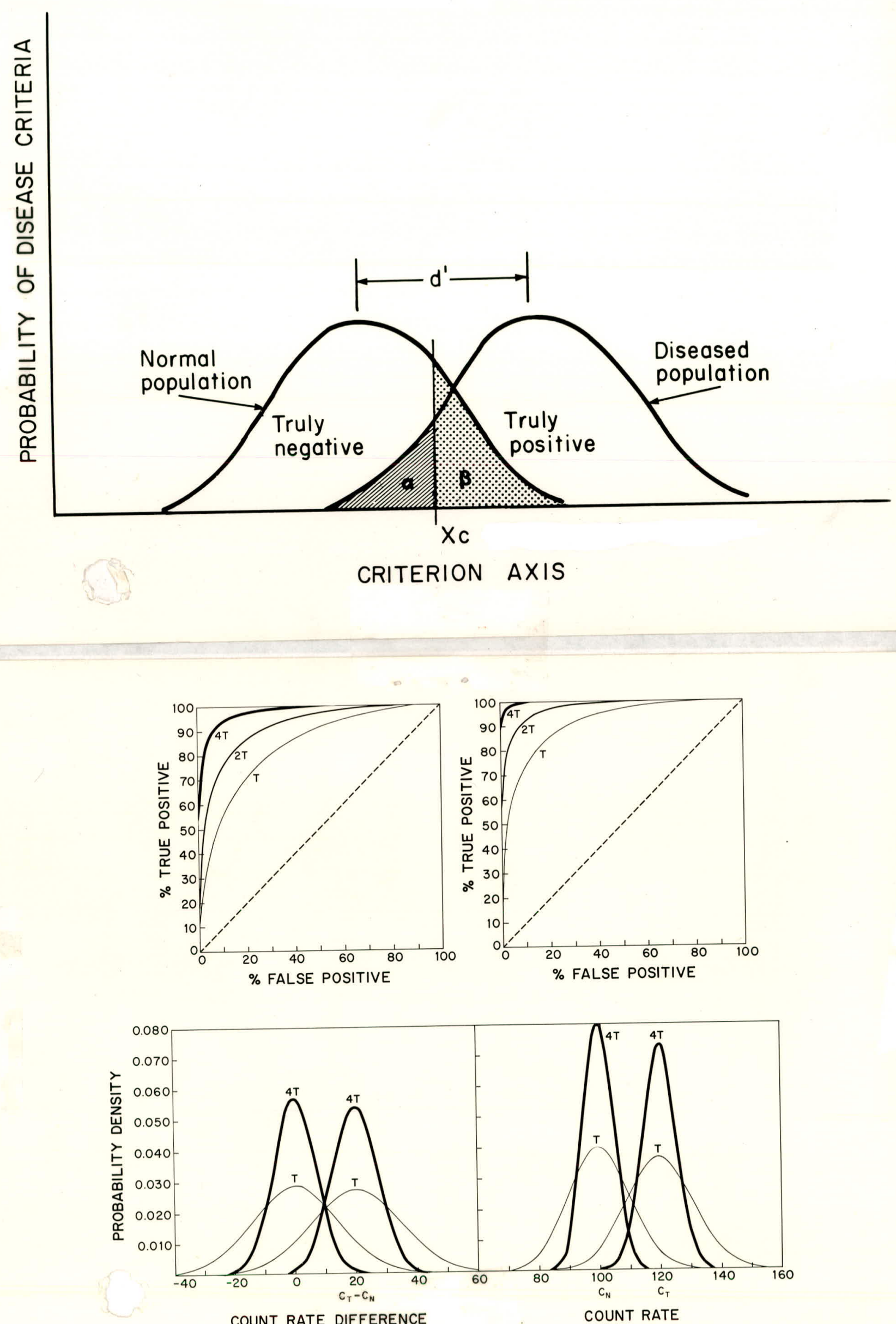

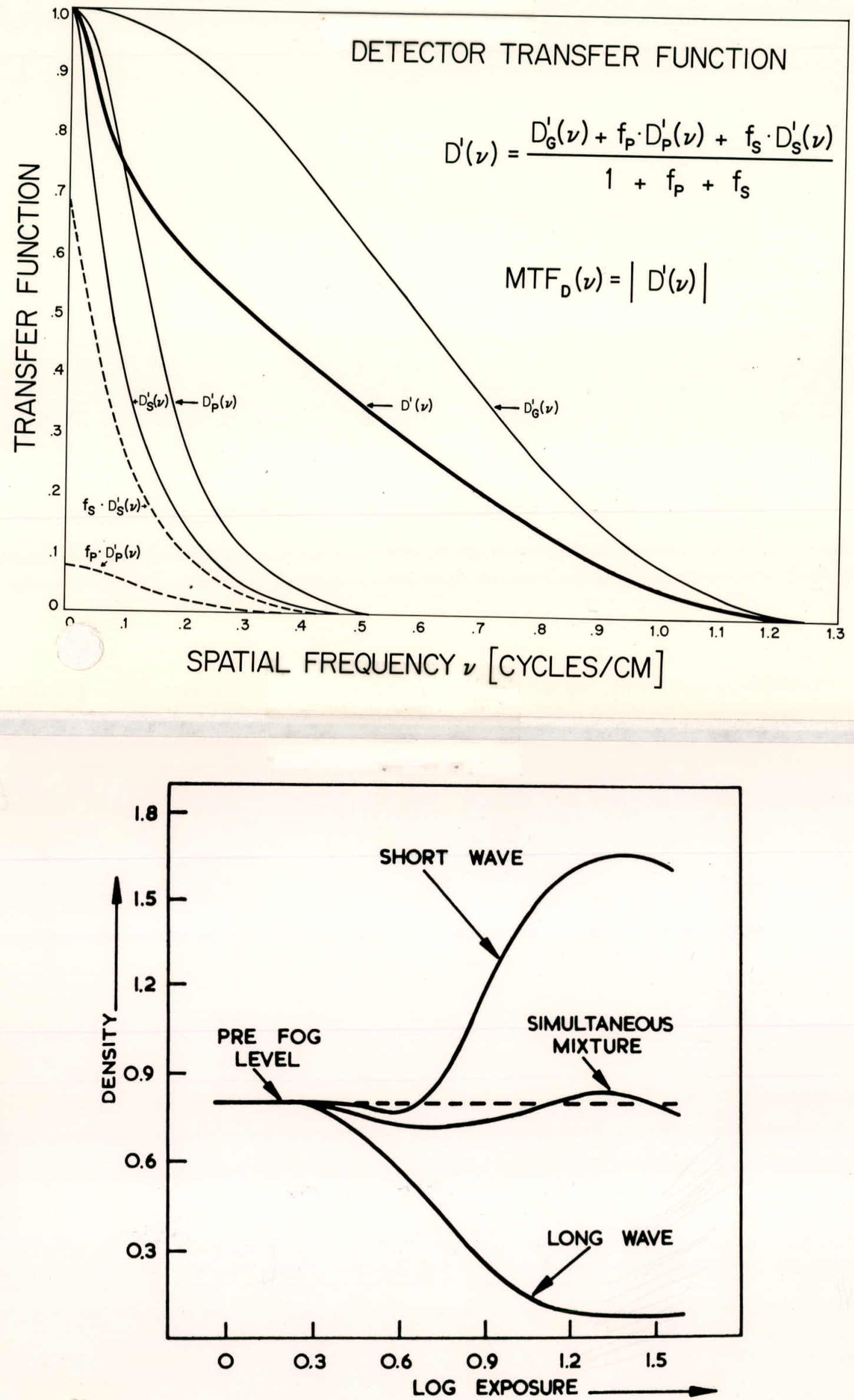